\title{
Integrated Computational Materials Science and Engineering of Textile Polymer Composites
}

\author{
Christian Heinrich*, Michael Aldridge ${ }^{\dagger}$, Alan S. Wineman ${ }^{\ddagger}$, John Kieffer ${ }^{\S}$ \\ and Anthony M. Waas $₫$ \\ University of Michigan, Ann Arbor, Michigan, 48109, USA
}

\begin{abstract}
An integrated computational framework for textile polymer composites is introduced. A novel polymer curing model is used in connection with modeling the manufacturing process of textile composites. The model is based on the notion of polymer networks that are continuously formed in a body of changing shape due to changes in temperature, chemistry and external loads. Nonlinear material behavior is incorporated through nonlocal continuum damage mechanics that preserves mesh objectivity in calculations that go beyond maximum loads. The integrated model is applied to the curing of plain weave textile composites made from carbon fiber tows and Epon ${ }^{\mathrm{TM}} 862$ resin. The mechanical and chemical properties are measured during curing using concurrent Brillouin and Raman light scattering. It is shown that significant stresses can develop during cure. The effect of these stresses on the manufactured part performance, when subsequent service loads are applied, is evaluated and a reduction in ultimate load, in agreement with experimental observations, is observed.
\end{abstract}

\section{Nomenclature}

$\begin{array}{ll}A & \text { Area, } \mathrm{m}^{2} \\ A_{d} & \text { Damaged area, } \mathrm{m}^{2} \\ A_{1}, A_{2} & \text { Frequency constants, } 1 / \mathrm{s} \\ \underline{C} & \text { Stiffness tensor, } \mathrm{Pa} \\ \overline{\overline{\bar{D}}} & \text { Damage } \\ D(t, s) & \text { Damage at time } \mathrm{t} \text { in network created at time } \mathrm{s} \\ E & \text { Elastic modulus, } \mathrm{Pa} \\ F & \text { Force, } \mathrm{N} \\ \Delta E_{1}, \Delta E_{2} & \text { Activation energy, } \mathrm{J} / \mathrm{mol} \\ H_{r} & \text { Heat of reaction, } \mathrm{J} \\ K & \text { Bulk modulus, } \mathrm{Pa} \\ M & \text { Plane wave modulus, } \mathrm{Pa} \\ R & \text { Gas constant, } \mathrm{J} / \mathrm{mol} / \mathrm{K} \\ T & \text { Temperature, } \mathrm{K} \\ T & \text { Traction, Pa } \\ \Delta V & \text { Activation volume, } \mathrm{m}^{3} / \mathrm{mol} \\ c & \text { Specific heat, } \mathrm{J} / \mathrm{kg} / \mathrm{K}\end{array}$

\footnotetext{
*Graduate Student Research Assistant, Department of Aerospace Engineering, 1320 Beal Avenue, 48109 Ann Arbor, MI, Student Member AIAA

${ }^{\dagger}$ Graduate Student Research Assistant, Department of Materials Science and Engineering, 2300 Hayward St, 48109 Ann Arbor, MI, Non-member

¥Professor of Mechanical Engineering, Department of Mechanical Engineering, 2350 Hayward, 48109 Ann Arbor, MI, Nonmember

§Professor of Materials Science and Engineering, Department of Materials Science and Engineering, 2300 Hayward St, 48109 Ann Arbor, MI, Non-member

๑Felix Pawlowski Collegiate Professor of Aerospace Engineering, Department of Aerospace Engineering, 1320 Beal Avenue, 48109 Ann Arbor, MI, Fellow AIAA
} 


$\begin{array}{ll}e & \text { Energy, J } \\ k_{1}, k_{2} & \text { Arrhenius type functions } \\ \ell & \text { Length scale parameter, } \mathrm{m} \\ m, n & \text { Fitting constants for curing law } \\ p & \text { Pressure, } \mathrm{Pa} \\ q_{i} & \text { Heat flux, } \mathrm{W} / \mathrm{m}^{2} \\ r & \text { Heat source, } \mathrm{W} \\ s & \text { Time of network creation, } \mathrm{s} \\ t & \text { Current time, } \mathrm{s} \\ x_{i} & \text { Spacial coordinate, m } \\ \alpha & \text { Linear coefficient of thermal expansion, } 1 / \mathrm{m} \\ \eta & \text { Parameter related to strain, used to find } \bar{\eta} \\ \bar{\eta} & \text { Nonlocal parameter } \\ \varepsilon_{i j} & \text { Strain } \\ \varepsilon_{c} & \text { Curing strain } \\ \kappa & \text { Thermal conductivity, W/m/K } \\ \mu & \text { Per-network shear modulus, Pa } \\ \phi & \text { Degree of cure } \\ \rho & \text { Density, kg/m }{ }^{3} \\ \sigma_{i j} & \text { Stress, Pa } \\ & \end{array}$

\section{Introduction}

Textile composites manufactured from carbon fiber tows and epoxy matrix are finding wide use in aerospace and other industrial applications. Such composites include 2D and 3D woven and braided composites comprised of glass or carbon fiber and polymer matrix. In woven composites, the fibers are combined into fiber tows consisting of thousands of individual fibers. These fiber tows are woven or braided using many different weaving patterns. Of interest in this study are 2 dimensional, triaxially braided composites (2DTBC). ${ }^{1-5}$ In 2DTBC, bias tows are braided at an angle to an axial tow. What makes woven composites an interesting material for engineering applications is their increased fracture toughness compared to prepreg and stitched composites due to the lack of a clear path for a macroscopic crack to progress. Due to an undulating textile architecture, cracks progressing in the matrix can be arrested at intersections of fiber tows. Thus, textile fiber composites are of great interest in the design of crashworthy vehicle structures.

Textile composites offer the added advantage that high volumes of parts can be manufactured at relatively reduced costs. In addition, textile preforms can be used for net-shape manufacturing which can lead to reduced part count. During the manufacturing of a composite part from textile fabrics, the dry fabric is placed in the mold and than the liquid resin is inserted into the mold afterwards using resin transfer molding (RTM) process. The wetting of the fibers may be aided with the help of vacuum. The corresponding process is called Vacuum Assisted Resin Transfer Molding (VARTM). Here, a vacuum is created inside the mold. Liquid epoxy resin is ejected through one or several ports on one side of the mold and pulled to other ports that are used to create the vacuum. RTM and VARTM can be performed at room temperature, thus eliminating the need for an oven and allowing to manufacture large parts such as wind turbine blades.

The design process with these materials remains challenging. Material properties need to be identified and the response up to and including failure needs to be investigated properly. During the characterization process of such a composite, Song et al. ${ }^{2}$ have noted that effective overall composite 2DTBC material properties are not in agreement with properties based on calculations with individual "virgin" constituent properties. The experimentally observed maximum service loads were found to be significantly lower compared to computational predictions. This disagreement has been attributed to changes in the state of the matrix due to curing in the presence of fibers. These changes are due to a mismatch in the thermal coefficient of expansion, cure shrinkage of the epoxy and thermal gradients causing different rates and degree of cure throughout the curing volume of matrix material. Stresses are present at the end of cure that may reduce the margin of load that can be applied to the part before the onset of inelastic response and failure. To capture the correct stress state of the resin inside the fully relaxed (no external load applied) composite, attempts have been made to measure the "in-situ" matrix properties. ${ }^{4,6}$ Here the material is treated to be stress free 
after cure and prior to loading, but effective or equivalent material properties are subsequently adopted. The "in-situ" properties are extracted using an inverse modeling approach. The idealized complex geometry of the textile composite is created in a finite element (FE) model. Then the material properties of the matrix material in the computational model are altered until the simulation results agree with the experimental results of a suitable coupon level test. Such an approach is suitable to investigate the response of a specific textile composite, and to obtain the "in-situ" material properties. However, this inverse modeling procedure to find the "in-situ" properties has to be repeated each time the properties of the textile composite and parameters of the curing process are changed.

In this paper, an integrated computational modeling approach that considers the textile composite manufacturing process and its subsequent service is presented. The only composite properties used in the numerical model is the geometry. Material properties of fiber and matrix material including the evolution of material properties of the matrix during cure are found from the constituents individually. As in previous studies that investigated the cure of epoxy resins, it is necessary to include various physical concepts to properly determine the importance of the various curing parameters. The curing of epoxy is a highly exothermic reaction $^{7-9}$ that can lead to appreciable temperature gradients in thick composites. The chemical evolution is tightly linked to the temperature and finally the evolution of stresses and material properties of the bulk composite depends on both the temperature history and curing history of the resin.

In developing a model for the cure generated stresses, the possibility of matrix failure during cure and prior to the onset of service load application must be considered. The computational models for failure prediction employed in the past can roughly be divided into two categories. On the one hand macroscopic cracks and failure have been captured through the use of discrete cohesive zone models (DCZM). ${ }^{10,11}$ These models usually require a-priori knowledge of the intended crack path. The other major model to capture failure is the continuum damage model $(\mathrm{CDM}) .{ }^{12,13}$ In CDM, micro cracks and voids are not explicitly modeled. Instead damage due to microstructural changes are accounted for in an average sense by degrading material properties. CDM models do not predict the formation of macroscopic cracks. On the other hand, when modeling failure with CDM, the location where damage will occur does not need to be known. Therefore, CDM within a nonlocal computational framework is employed to model failure of epoxy during and after cure.

Finally, it is noted that the curing process can produce self-equilibrating internal stresses within the material. If the process is modeled and understood properly, then the desired internal stress state can be "designed" so that subsequent part performance is optimized. Such an approach has precedent in the manufacturing process of auto-glass, where compressive surface stresses are "built-in" by tailoring the manner by which the cooling process is controlled, as shown in the pioneering work by Lee et al. ${ }^{14}$ In that work, it is shown that different cooling rate histories can produce undesired surface tensile stresses, which if present, can accelerate the propagation of surface flaws like cracks, when tensile service loads are experienced. In this paper, a novel model is introduced to capture the effects of the manufacturing process on the state of the cured matrix within a plain weave composite. This model is used to evaluate the mechanical response of the plain weave composite when subjected to service loads. The maximum applicable service load is dependent on the curing related properties of the polymer matrix material. The holistic computational modeling procedure introduced here (manufacturing and subsequent service loads) shows the need for an approach that integrates materials science and engineering, leading to the title of this paper - "integrated computational materials science and engineering of textile polymer composites".

\section{A. Related Past Work}

\section{Mechanical properties of textile composites}

The mechanical properties of textile composites have been extensively studied in the past and a summary can be found in the work by Song. ${ }^{1}$ Early investigations were aimed mostly at predicting elastic properties of plain weave composites. ${ }^{15-19}$ The onset of inelastic response of plain weave composites was considered by Karkkainen and Sankar. ${ }^{20}$ They constructed the failure envelope of a plain weave woven unit cell and noted a close relation to the Tsai-Wu failure criterion. ${ }^{21}$ Whitecomb and Srirengan ${ }^{22}$ investigated progressive damage of plain weave composite. They used a static finite element simulation to determine the locations of largest stress and failure onset. At these points the material properties were modified and a new equilibrium state was calculated. This process was repeated until no further damage occurred and the global strain was increased until the next onset of damage. 
Recently, the elastic properties and failure response of 2D triaxial braided composites (2DTBC) received considerable attention. Quek et al. ${ }^{23}$ developed an analytical model to predict the stiffness of 2DTBC. Each tow was first modeled as a transversely isotropic material, where the effective elastic material properties were determined using the concentric cylinder model (CCM). Then the undulating tows were idealized as sinusoidal waves. To calculate compression response, a finite element analysis of a representative unit cell was performed that included both, geometric and material non-linearities. ${ }^{24}$ The fiber material was treated as an elastic material and the epoxy matrix was modeled using elastic-plastic stress-strain relations and incremental $\mathrm{J}_{2}$-flow theory of plasticity. Song and co-workers investigated the compressive response of 2DTBC experimentally and through finite element simulations. ${ }^{1-5}$ The finite element model used was of an idealized representative unit cell (RUC) consisting of fiber tow and matrix. The fibers were treated as elastic and the matrix material was treated as plastic or plastic-damaging. The resulting nonlinear fiber tow behavior was modeled using Hill's theory of anisotropic plasticity. ${ }^{25}$

\section{Cure of epoxy resin and composites}

Manufacturing induced effects have usually been studied in the context of cure induced stresses and potential failure during cure. Rabearison et al. ${ }^{26}$ give an example of how gradients in cure develop in a thick carbon epoxy tube and subsequently cause defects such as cracks. Rabearison et al. than developed a numerical model for curing of an epoxy. They calculate the curing of the epoxy in a glass beaker and conclude that stresses large enough to cause cracks are developed during the curing process. In a similar fashion Corden et al. ${ }^{27}$ have developed a curing model to predict the residual stresses in thick resin transfer molded laminated cylinders. They identified chemical shrinkage due to cure as a major contributing mechanism for stress build up as they cured all parts at room temperature. During the experimental validation of the results with different laminate layouts it was noted that interlaminar cracking can occur due to differential shrinkage. Plepys and Ferris ${ }^{28}$ noted the creation of cracks during isothermal cure of three-dimensionally constrained epoxy resin in a glass cylinder. Chekanov et al. ${ }^{29}$ have identified different defect types that may occur during the constrained cure of epoxy resins. At high temperature, cohesive defects intersecting the resin were observed, at medium temperatures uneven voids were found and at low temperatures zigzag defects and cohesive smaller defects were seen.

Besides stress generation and possible cracking during cure, geometric tolerances are another important issue that needs to be considered. For example, a thick composite may be cured on a metal tool with two perpendicular side faces to give an L-shaped composite part. Upon removal of the part from the mold, a "spring-in" can be observed. Here, the two sides of the L-shaped part bend inwards giving a smaller angle than the anticipated $90^{\circ} \cdot{ }^{30-33}$ Equivalently, when a composite part is cured on a flat tool, bending or warpage can be observed due to thermal gradients and residual stresses build up during cure. ${ }^{34-36}$ The dimensional accuracy and stress generation in woven composites has been studied in the context of woven circuit boards. ${ }^{37}$ Proper knowledge of curing induced distortion can be used to design a mold that will compensate for such effects, as demonstrated by Capehart et al. ${ }^{38}$

Studies that investigate the curing process of composites are commonly broken down into two parts: chemical reaction, heat generation and heat conduction, and, the evolution of stress and development of structural integrity. In order to understand and optimize the manufacturing process both issues must be understood well. The temperature field is usually modeled using the standard heat equation and Fourier's law. The cure kinetics are usually modeled using a phenomenological model proposed by Kamal. ${ }^{39}$ The evolution of stress on the other hand is modeled through a variety of approaches. Plepys and Farris ${ }^{28}$ and Plepys et al. ${ }^{40}$ used incremental elasticity to describe the evolution of stresses and elastic properties as a function of cure. They assume that during each time increment, the stress increment is related to the strain increment as a linear isotropic elastic relation with a changing modulus as a function of cure. Bogetti and Gillespie $^{41}$ used a linear mixing rule based on degree of cure, elastic modulus of uncured and fully cured resin moduli and a parameter to quantify stress relaxation and chemical hardening. Another material model often used involves linear viscoelasticity. ${ }^{42-45}$ Here the evolution of the cure dependent and therefore time dependent material properties was modeled with the aid of integral constitutive equations found in linear viscoelasticity. A more sophisticated model has been proposed by Adolf and Chambers ${ }^{46}$ using non-linear viscoelasticity. However, such a model with its large amount of parameters also requires extensive validation and experimental investigation. ${ }^{47}$ The approach that will be used in this study is based on previous work by Mei et al. ${ }^{48}$ and Mei, ${ }^{49}$ who investigated solidification of epoxies using a novel network forming model where networks are continuously created in a new reference configuration as cure progresses and each network had 
different visco-elastic material properties.

With the different curing and stress generation models presented, various studies have been performed to optimize aspects of the curing cycle. Li et al. ${ }^{50}$ solved a coupled thermal and chemical problem to investigate an optimal curing cycle for thermoset matrix composites that was optimized for low curing time, but limiting the maximum temperature found in the epoxy. White and $\mathrm{Hahn}^{36}$ optimized the curing process to reduce the residual stress in a composite part using a three-step curing cycle. Gopal et al. ${ }^{51}$ also aimed for a reduction in residual stress, but they only used a two step curing cycle. Finally, Zhu and Geubelle ${ }^{52}$ investigated the sensitivities of the curing process parameters on the dimensional accuracy of L-shaped composite parts.

\section{Material Model}

The specific material of interest is a $2 \mathrm{DTBC}$ made of Epon ${ }^{\mathrm{TM}} 862$ resin and carbon fibers. During the manufacturing process, the un-wetted fibers are placed in a mold and a resin-hardener mixture is applied. The mold is then compressed and kept at a prescribed boundary temperature. To fully understand the manufacturing process, several physical effects have to be taken into consideration.

\section{A. Heat Conduction and Curing}

The curing of an epoxy is a highly exothermic chemical reaction (see, for example, studies regarding DSC of epoxy ${ }^{7-9}$ ). The degree of cure is often measured by placing a small sample in a digital scanning calorimeter, maintaining the sample at a constant temperature and measuring the heat that is generated during cure. Denote the heat generated by $H(t)$ and the degree of cure by $\phi(t)$, both at time $t$. The degree of cure is defined by ${ }^{39}$

$$
\phi(t)=\frac{H(t)}{H_{r}},
$$

where $H_{r}$ is the total heat that is generated. Thus, $\phi(t)$ monotonically increases from $\phi=0$, at the uncured state, to $\phi=1$, at the fully cured state. Note that the rate of heat generation per unit mass is

$$
r=\frac{\mathrm{d}\left(H_{r} \phi\right)}{\mathrm{d} t}
$$

The process is modeled by a curing kinetics equation of the form

$$
\frac{\mathrm{d} \phi}{\mathrm{d} t}=f(T, \phi),
$$

where $T$ is the temperature and $f(T, \phi) \geq 0 . \mathrm{Kamal}^{39}$ has suggested that $f(T, \phi)$ be given by,

$$
\begin{array}{r}
f(T, \phi)=\left(k_{1}(T)+k_{2}(T) \phi^{m}\right)(1-\phi)^{n}, \\
k_{1}(T)=A_{1} \exp \left(-\frac{\Delta E_{1}}{T R}\right), \\
k_{2}(T)=A_{2} \exp \left(-\frac{\Delta E_{2}}{T R}\right),
\end{array}
$$

in which $m$ and $n$ are constants, $R$ is the gas constant, $A_{1}, A_{2}$ are frequency like constants and $\Delta E_{1}, \Delta E_{2}$ are activation energies. Choices for $m, n, k_{1}(T)$ and $k_{2}(T)$ for various epoxy-curing agent systems have appeared in the literature. In applications, equation (3) is used to describe the curing process even when the temperature varies with time. ${ }^{9}$

In a curing structural composite, there is both local heat generation due to curing and heat conduction due to the presence of an external surface or along fibers. This process is governed by the local form of the first law of thermodynamics, ${ }^{53,54}$

$$
\rho \frac{\mathrm{d} e}{\mathrm{~d} t}=-\frac{\partial q_{i}}{\partial x_{i}}+\rho r+\sigma_{i j} \frac{\mathrm{d}}{\mathrm{d} t} \varepsilon_{i j}
$$


where $e$ is the internal energy per unit mass, $\rho$ is the current mass density, $q_{i}$ are the components of the heat flux vector, $r$ is the rate of heat supply per unit mass, $\sigma_{i j}$ are the stress components and $\varepsilon_{i j}$ are the components of the infinitesimal strain tensor.

For the applications considered here, the rate of mechanical work, $\sigma_{i j} \mathrm{~d} \varepsilon_{i j} / \mathrm{d} t$, is assumed to be negligible and the internal energy is assumed to be proportional to temperature, $e=c T$. The heat flux vector is related to the temperature gradient by the Fourier law of heat conduction,

$$
q_{i}=-\kappa \frac{\partial T}{\partial x_{i}},
$$

where $K$ is the thermal conductivity. It is possible that the thermal conductivity depends on the degree of cure and the temperature, i.e. $K=K(T, \phi)$. Equation (7), with (2), (8) and these assumptions, becomes

$$
\rho c \frac{\partial T}{\partial t}=\frac{\partial}{\partial x_{i}}\left(\kappa(\phi, T) \frac{\partial T}{\partial x_{i}}\right)+\rho H_{r} \frac{\partial \phi}{\partial t} .
$$

Equations and (3),(4) and (9) form a system of coupled nonlinear partial differential equations for the spatial distribution and time variation of temperature, $T(\underline{x}, t)$, and degree of cure, $\phi(\underline{x}, t)$.

\section{B. Stress Development}

To develop an understanding of the stresses and deformations during polymer cure, a network model of a polymer will be adopted. The networks interpenetrate each other, i.e. they occupy the same volume. On the other hand the assumption is made that they do not interact with each other.

A second assumption is related to the interaction of the different physical effects mentioned earlier: heat conduction, chemical change and mechanical response. Curing of epoxy resins is often done under pressure. This has influence on the rate of cure and can be taken into account. Ramos et al. ${ }^{55}$ measured and modeled the cure kinetics and shrinkage for a DGEBA/ MCDEA epoxy system. The reaction rate was investigated over a range of 200-600 bar pressure and a shift in reaction rate was noticeable. For this kind of analysis, where the stress and chemical composition depend on each other, the term mechanochemistry has been used in the past. ${ }^{56}$ Ramos et al. ${ }^{55}$ suggested to augment equations (5) and (6) such that:

$$
k_{i}(T, p)=A_{i 0} \exp \left(-\frac{\Delta E_{1}}{T R}\right) \exp \left[\left(\frac{-\Delta V}{T R}+\frac{1}{V} \frac{\partial V}{\partial p}\right)\left(p-p_{0}\right)\right], \quad i=1,2
$$

where $\Delta V$ is the activation volume and $p$ is the pressure. The dependency of rate of cure on temperature is significantly stronger than on pressure in the range 1-140 bar, which is observed during the curing process in the mold. ${ }^{57-59}$ Therefore, the dependency on pressure will be ignored. It is also desirable to separate the thermo-chemical problem from the mechanical problem from a numerical point of view. This leads to a one way coupling. The temperature and chemical composition are independent of stress state but the stress state depends on the degree of cure through evolving material properties and on temperature through thermal expansion.

In what follows, a model for stress evolution that incorporates the notion of networks forming and contributing to evolving stiffness is derived for a $1 \mathrm{D}$ model and then generalized to three dimensions. The creation of the epoxy networks as curing evolves is depicted in figure $1{ }^{49}$ Initially, hardener and epoxy resin are in a liquid state. They are mixed and poured into a mold with the fibers. Immediately after mixing, the pure epoxy resin starts reacting, building connections and cross links. At some time $t_{1}$ the first network forms. It is assumed, that it forms in a stress free configuration. The forming of the network is accompanied by some shrinkage due to cure. There also might be additional strain due to changes in temperature and externally applied loads. At some later time, $t_{2}$, another network forms. This network has a different stress free reference configuration. Even later, a new network forms at time $t_{3}$. Its stress free reference configuration is different than the one from the networks formed at time $t_{1}$ and $t_{2}$. Because all networks occupy the same volume, their current configuration is the same. On the other hand their stress free reference configuration is different. As a result, the stress state in each network is different. The sum of all stresses within the networks is such that it balances out all externally applied stresses.

Figure 2 depicts the formation of networks as one dimensional springs. The derivation is similar to the ones given by $\mathrm{Mei}^{49}$ and Hossain et al. ${ }^{60}$ A network forms at time $t_{1}$ and might experience some external strain due to external load. The response of the network is assumed to be elastic. The stress $\sigma(t, s)$ at time 
configuration
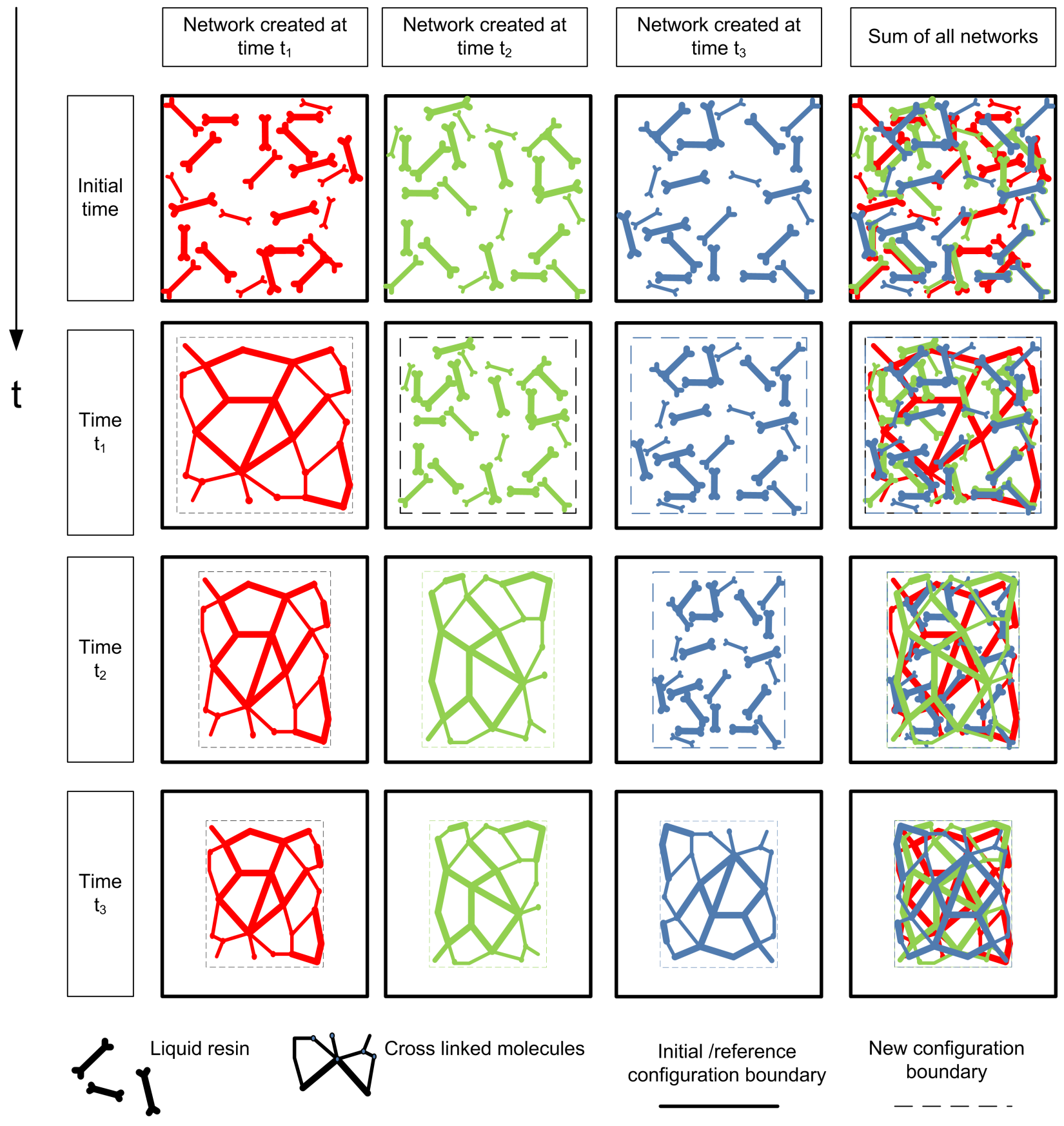

Initial/reference
configuration boundary

New configuration boundary

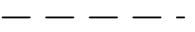

Figure 1. A schematic of the forming of networks as curing evolves 
$t$ in a network created at time $s$ is proportional to the mechanical strain $\varepsilon^{m e c h}(t, s)$ relative to its stress free reference configuration multiplied with the elastic modulus $E(s)$ associated with that network.

$$
\sigma(t, s)=E(s) \varepsilon^{m e c h}(t, s)
$$

The total strain at time $t$ in the network formed at time $s$ is the sum of all incremental strains since its creation. This is the same as the mechanical strain including thermal effects and shrinkage effects. $\Delta \varepsilon\left(t_{i}\right)$ is the increment in total strain from time $t_{i-1}$ to time $t_{i}$.

$$
\begin{aligned}
\varepsilon(t, s) & =\Delta \varepsilon(s)+\Delta \varepsilon(s+\Delta t)+\cdots+\Delta \varepsilon(t) \\
& =\varepsilon^{\text {mech }}(t, s)+\alpha(s) \Delta(T(t)-T(s))-\varepsilon_{c}(s)
\end{aligned}
$$

Here, $\varepsilon_{c}(s)$ is the cure shrinkage strain of the network formed at time s. The sign of $\varepsilon_{c}(s)$ is positive when it decreases the strain. The stress in network 1 created at time $t_{1}$ is given by:

$$
\sigma\left(t_{1}, t_{1}\right)=E\left(t_{1}\right)\left(\Delta \varepsilon\left(t_{1}\right)+\varepsilon_{c}\left(t_{1}\right)-\alpha\left(T\left(t_{1}\right)-T\left(t_{1}\right)\right)\right)
$$

Curing strains begin to impact each network, after it is formed. Strains due to external loads and temperature differences have to be accounted for at all times. The stresses in network 1 and 2 at the formation time of network 2 are given by:

$$
\begin{aligned}
\sigma\left(t_{2}, t_{1}\right) & =E\left(t_{1}\right)\left(\Delta \varepsilon\left(t_{1}\right)+\Delta \varepsilon\left(t_{2}\right)+\varepsilon_{c}\left(t_{1}\right)-\alpha\left(T\left(t_{2}\right)-T\left(t_{1}\right)\right)\right) \\
\sigma\left(t_{2}, t_{2}\right) & =E\left(t_{2}\right)\left(\Delta \varepsilon\left(t_{2}\right)+\varepsilon_{c}\left(t_{2}\right)-\alpha\left(T\left(t_{2}\right)-T\left(t_{2}\right)\right)\right.
\end{aligned}
$$

Furthermore, in the next step, the total strains of the reference configuration change by the amount $\Delta \varepsilon_{3}$ after network 3 is created:

$$
\begin{aligned}
\sigma\left(t_{3}, t_{1}\right) & =E\left(t_{1}\right)\left(\Delta \varepsilon\left(t_{1}\right)+\Delta \varepsilon\left(t_{2}\right)+\Delta \varepsilon\left(t_{3}\right)+\varepsilon_{c}\left(t_{1}\right)-\alpha\left(T\left(t_{3}\right)-T\left(t_{1}\right)\right)\right) \\
\sigma\left(t_{3}, t_{2}\right) & =E\left(t_{2}\right)\left(\Delta \varepsilon\left(t_{2}\right)+\Delta \varepsilon\left(t_{3}\right)+\varepsilon_{c}\left(t_{2}\right)-\alpha\left(T\left(t_{3}\right)-T\left(t_{2}\right)\right)\right) \\
\sigma\left(t_{3}, t_{3}\right) & =E\left(t_{3}\right)\left(\Delta \varepsilon\left(t_{3}\right)+\varepsilon_{c}\left(t_{3}\right)-\alpha\left(T\left(t_{3}\right)-T\left(t_{3}\right)\right)\right)
\end{aligned}
$$

Each network is weighted to contribute to the total load carrying capability in a "rule of mixtures" sense. During the creation of each network, the overall epoxy has cured by an amount of $\Delta \phi\left(t_{i}\right)=\phi\left(t_{i}\right)-\phi\left(t_{i-1}\right)$, where, $\phi$ denotes the degree of cure as defined by equation (1). In the following, it will be assumed that an equivalent interpretation of the degree of cure $\phi$ is given by

$$
\begin{aligned}
\phi(t) & =\frac{\text { mass cured at time } t}{\text { total mass of material that can cure }} \\
& =\text { mass fraction of cured material }
\end{aligned}
$$

$$
\mathrm{d} \phi=\text { mass fraction cured during the time interval from } s \text { to } s+\mathrm{d} s
$$

The total stress in the epoxy at time $t_{N}$ is given by:

$$
\sigma_{t o t}\left(t_{N}\right)=\sum_{i=1}^{N} \Delta \phi\left(t_{i}\right) \sigma\left(t_{N}, t_{i}\right)=\sum_{i=1}^{N} \Delta \phi\left(t_{i}\right) E\left(t_{i}\right)\left(\varepsilon_{c}\left(t_{i}\right)-\alpha\left(T\left(t_{N}\right)-T\left(t_{i}\right)\right)+\sum_{j=i}^{N} \Delta \varepsilon\left(t_{N}, t_{j}\right)\right)
$$

The tot subscript will be omitted in the following, when it is clear that the total stress is meant. When taking the limit of the above equation, the sums can be converted into integrals:

$$
\sum \Delta \phi \rightarrow \int \mathrm{d} \phi \text { and } \sum \Delta \varepsilon \rightarrow \int \mathrm{d} \varepsilon
$$




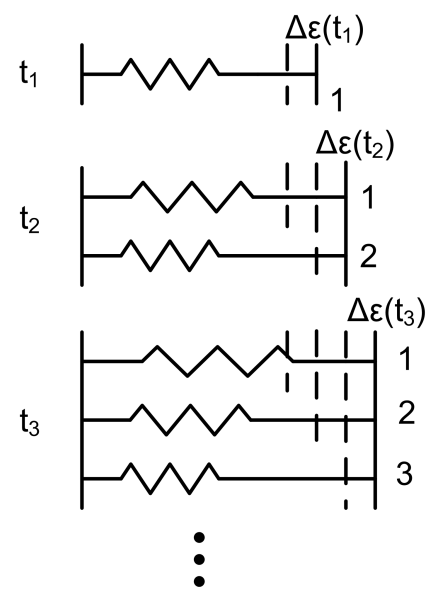

Figure 2. Forming of 1D networks, shown schematically

The discrete quantity $\varepsilon_{c}\left(t_{i}\right)$ represents the curing strain in the $i^{t h}$ network formed at time $t_{i}$. It's continuous equivalent is $\varepsilon_{c}(s)$, the cure shrinkage in the network formed at time $s$. Equivalent expressions exist for the material properties $E\left(t_{N}\right) \rightarrow E(s)$ and $\alpha\left(t_{N}\right) \rightarrow \alpha(s)$. With these substitutions, the stress up to a certain amount of cure, represented by the cure fraction, $\Phi$ is given by:

$$
\begin{aligned}
\sigma(\Phi) & =\int_{0}^{\Phi} \tilde{E}(\phi)\left(\varepsilon_{c}(\phi)-\alpha(T(\Phi)-T(\phi))+\int_{\varepsilon(\phi)}^{\varepsilon(\Phi)} \mathrm{d} \bar{\varepsilon}\right) \mathrm{d} \phi \\
& =\int_{0}^{\Phi} \tilde{E}(\phi)\left(\varepsilon_{c}(\phi)-\alpha(T(\Phi)-T(\phi))+\varepsilon(\Phi)-\varepsilon(\phi)\right) \mathrm{d} \phi
\end{aligned}
$$

The degree of cure $\phi(s)$ increases monotonically with time which allows for the substitution, $\mathrm{d} \phi=\frac{\mathrm{d} \phi}{\mathrm{d} s} \mathrm{~d} s$ :

$$
\sigma(t)=\int_{0}^{t} \frac{\mathrm{d} \phi}{\mathrm{d} s} E(s)\left(\varepsilon(t)-\varepsilon(s)+\varepsilon^{c u r e}(s)-\alpha(T(t)-T(s))\right) \mathrm{d} s
$$

The elastic modulus $E(s)$ depends directly on the degree of cure, and indirectly on time, by $E(s)=\tilde{E}(\phi(s))$. The above equation can be generalized to three dimensions:

$$
\begin{aligned}
\underline{\underline{\sigma}}= & \int_{0}^{t} \frac{\mathrm{d} \phi}{\mathrm{d} s} \underline{1}\left[K(s) \operatorname{tr}\left(\underline{\underline{\varepsilon}}(t)-\underline{\underline{\varepsilon}}(s)+\underline{\underline{\varepsilon_{c}}}(s)-\underline{\underline{1}} \alpha(s) \Delta T(t, s)\right)\right. \\
& \left.+2 \mu(s)\left(\underline{\underline{\varepsilon}}(t)-\underline{\underline{\varepsilon}}(s)+\underline{\underline{\varepsilon_{c}}}(s)-\underline{\underline{1}} \frac{1}{3} \operatorname{tr}\left(\underline{\underline{\varepsilon}}(t)-\underline{\underline{\varepsilon}}(s)+\underline{\underline{\varepsilon_{c}}}(s)\right)\right)\right] \\
& +(1-\phi(t)) K_{l i q} \operatorname{tr}\left(\underline{\underline{\varepsilon}}(t)-\underline{\underline{1}} \alpha_{l i q} \Delta T(t)\right) \underline{\underline{1}}
\end{aligned}
$$

where $K$ is the per-network bulk modulus, $\mu$ is the per-network shear modulus and $\alpha$ is the linear coefficient of thermal expansion of each network. The notation, (.) indicates a second order tensor. The last term in the equation is due to the fact that a liquid can hold compressive volumetric stresses.

The tensor quantity $\underline{\underline{\varepsilon}}_{c}(s)$ describes the cure shrinkage strains of each network. It is assumed that curing only produces normal strains.

$$
\underline{\underline{\varepsilon_{c}}}(s)=\left[\begin{array}{ccc}
\varepsilon_{c}(s) & 0 & 0 \\
0 & \varepsilon_{c}(s) & 0 \\
0 & 0 & \varepsilon_{c}(s)
\end{array}\right]
$$


$\underline{\underline{\varepsilon}}_{c}(s)$ can be chosen such that macroscopically, a linear shrinkage as a function of degree of cure can be observed.

It is noted that formally $\alpha(s), \mu(s), K(s)$ are functions of time. But actually they are a function of cure, which has to be related to time, so that $\alpha(s)=\tilde{\alpha}(\phi(s)), \mu(s)=\tilde{\mu}(\phi(s)), K(s)=\tilde{K}(\phi(s))$ and $\varepsilon_{c}(s)=\tilde{\varepsilon}_{c}(\phi(s))$. These material functions can be determined from experiments.

\section{Development of damage}

The material response developed thus far is linear elastic upon full cure. It can therefore serve as a guide to curing conditions that will lead to small or large residual stresses, but it lacks the capability of predicting non-linear response during cure or subsequently in service. It will be shown that the epoxy in a composite can experience large stresses that can lead to damage in the form of cracks during the curing process. ${ }^{26,28}$ This is also depicted in figure 3, which shows a cross section image of a carbon fiber and Hetron ${ }^{\mathrm{TM}}$ resin tow. ${ }^{61}$ There is damage already present in the form of cracks in the composite just after cure and prior to application of mechanical loads. Therefore, it is important to extend the model to allow for the possibility of damage (non-reversible effects) to occur during the process of curing.

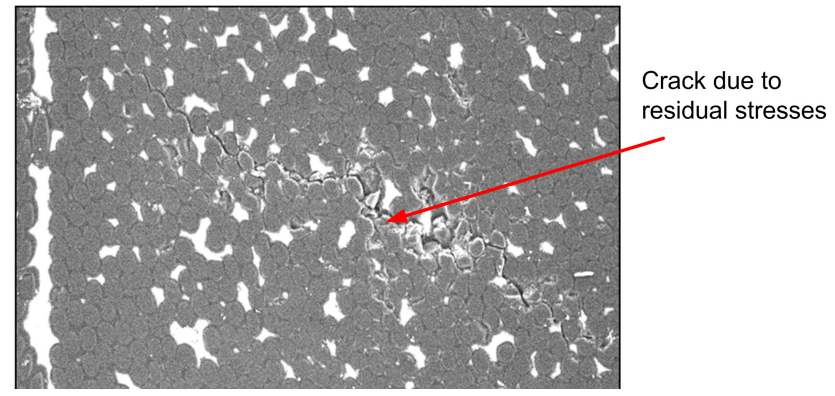

Figure 3. Crack in composite due to excessive stresses during cure

\section{Damage in continuum mechanics}

There is a multitude of ways to incorporate damage and failure into a material model. Classical small strain plasticity offers a convenient framework but with several drawbacks. The unloading slope of a plastically deformed "classical" solid will be proportional to the loading slope, as shown in figure 4(a). That means, the elastic properties are not altered in classical plasticity. Also, classical plasticity models are usually not capable of "ending" a stress-strain law. That means, continued loading will not lead to a decrease in stress, but will lead to continued flow, either with increasing stress (hardening) or constant stress (prefect plasticity). The most important consequence of damage is the reduction in stiffness (and/or stress) with continued deformation. Models that incorporate reduction in stress beyond a peak stress are termed "softening" stressstrain laws. Naturally, these laws will need to accommodate a negative tangent stiffness in the stress-strain response as schematically shown in figure $4(\mathrm{~b})$.

The physical significance of continuum damage mechanics can be explained both for metals and for polymers. ${ }^{62}$ In metals the damaging process might be regarded as the nucleation of microscopic cracks and voids. With increasing strain these will grow, coalesce and eventually form a macroscopic crack. In rubbery polymers on the other hand, damage can be seen as the rupture of polymer chains. Continued damage leads to continued breakage of bonds and therefore loss of stiffness. The earliest work in the area of continuum damage mechanics is usually attributed to Kachanov (the translation can be found in ${ }^{63}$ ) who introduced an internal variable to model creep failure. Rabotnov ${ }^{64}$ is attributed to giving physical meaning to the internal variable, although there has been some debate about whether such a thing should be attempted. ${ }^{65}$ Let us assume that damaged and undamaged load bearing cross sectional areas of a body are given by $A$ and $A_{d}$ respectively as shown in figure $6 .{ }^{66}$ Then the damage can be viewed as

$$
D=\frac{A-A_{d}}{A}
$$

If $D=0$, the material is damage free. $D=1$ corresponds to a fully damaged or ruptured body, i.e. it is broken into two parts. Thus, $0<D<1$, characterizes a partially damaged state. If one considers the force 

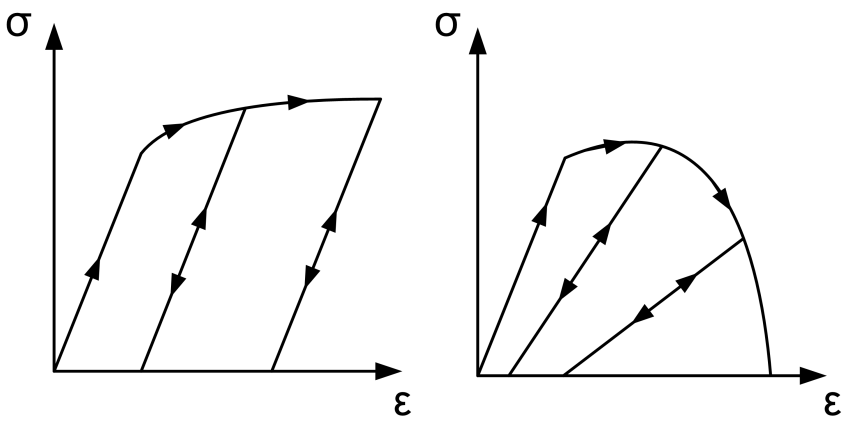

(a) Plastic material behavior

(b) Softening material behavior, including damage and plasticity

Figure 4. Comparison of plastic and damaging material behavior

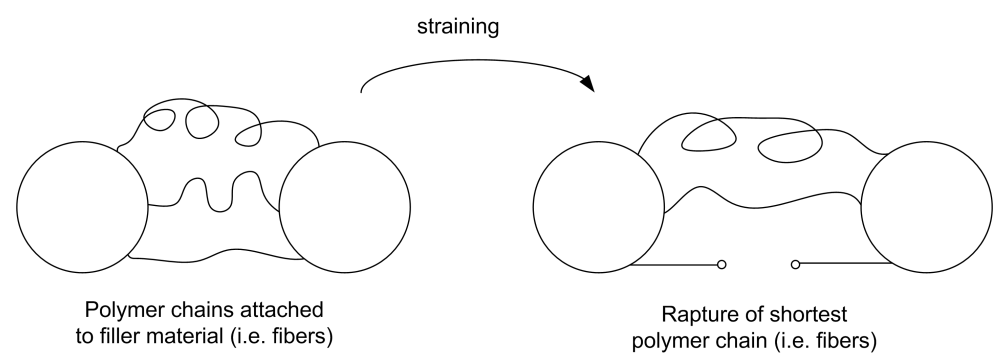

Figure 5. Damage of polymer chains ${ }^{62}$

11 of 33 
$\underline{F}$ acting normal to the surface $A$, the traction is given as $\underline{T}=\underline{F} / A \cdot{ }^{67}$ Then, an effective traction is defined by

$$
\underline{T}_{e f f}=\frac{\underline{F}}{A_{e f f}}=\frac{\underline{T}}{1-D}
$$

which leads, by the usual definition of stress and traction, $\underline{T}_{e f f}=\underline{\underline{\sigma}} \cdot \underline{n}$, to the effective stress

$$
\underline{\underline{\sigma}}_{e f f}=\frac{\underline{\sigma}}{1-D} \text {. }
$$

Using the linear elastic constitutive behavior, the relation between the stress and strain in the undamaged and damaged material, respectively are given by

$$
\begin{aligned}
\underline{\underline{\sigma}} & =\underline{\underline{\underline{\underline{C}}}}: \underline{\underline{\varepsilon}} \\
\underline{\underline{\sigma}}_{e f f} & =\underline{\underline{\underline{\underline{C}}}}_{e f f}: \underline{\underline{\varepsilon}}
\end{aligned}
$$

This leads to the effective stiffness

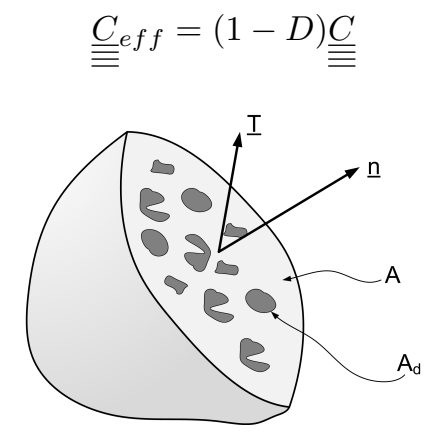

Figure 6. The effective stress concept ${ }^{13}$

With that, the final constitutive equation is given by

$$
\underline{\underline{\sigma}}=(1-D) \underline{\underline{\underline{\underline{C}}}}: \underline{\underline{\varepsilon}}
$$

It should be noted that implicitly the assumption has been made that damage can be described by a scalar value. Furthermore, this scalar measure of damage $D$ does not need to be monotonically varying with stress and/or strain. For example, it can be specified as a function of both, stress and strain in the form of a lookup table based on experimental observation. However, in this work it is assumed that the values of $D$ must conform to a reduction in stiffness with continued loading. That means, material healing is not allowed. Since $D$ is a scalar, the hypothesis of isotropy has been used. It simplifies calculations significantly but higher order expressions have been developed before. ${ }^{13}$

\section{Strain softening behavior}

In what follows, the material constitutive law, (32) will be used. That is, it will be assumed that a uniaxial stress-strain curve of the type shown in figure 7 has been provided as material data. It will be assumed that the post-peak response is solely due to damaging behavior. Thus, the model development will be for an elastic-damaging solid, whose uniaxial stress-strain response is described in figure 7.

It is often pointed out that a negative slope in the post-peak regime is physically not possible. It implies an imaginary speed of sound. ${ }^{68}$ However it can be argued that the observed result is the combined response of structural changes of a representative volume of material in which voids and cracks form in a distributed manner. An imaginary speed of sound is not the only problem intrinsic to this stress-strain formulation. A loss of objectivity can be observed in numerical calculations that adopt any type of discretization scheme. ${ }^{68}$ When calculating a solution using finite element methods, the result will be strongly dependent on the mesh size. ${ }^{54,68,69}$ Here, we propose a nonlocal formulation that provides mesh objectivity in the numerical solution. 


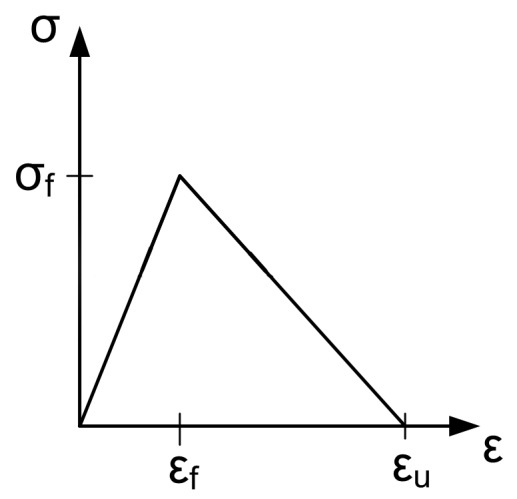

Figure 7. Softening stress-strain curve

\section{Nonlocal material model - formulation}

Since the response of a body that exhibits strain softening behavior cannot be successfully calculated using a standard finite element approach, a non-standard approach that uses a non-local material model is adopted. In non-local models, it is assumed that the material response at a point is not only a function of the state at that point, but also of the state of surrounding points. In nonlocal theories, the field quantities describing the material response are replaced by their nonlocal equivalent through a volume averaging procedure, as,

$$
\bar{f}(\underline{x})=\frac{1}{V} \int_{V} w(\underline{x}-\underline{\xi}) f(\underline{\xi}) \mathrm{d} \underline{\xi}
$$

Common choices for the weight function, $w(\underline{x}-\underline{\xi})$, include the Gauss distribution, the bell function ${ }^{70}$ and Green's function. ${ }^{71}$ They are shown in figure 8 , and stated below.

$$
\begin{aligned}
w_{\text {Gauss }} & =\exp \left(-\frac{n_{\text {dim }} r^{2}}{2 \ell^{2}}\right) \\
w_{\text {Bell }} & = \begin{cases}\left(1-\frac{r^{2}}{R^{2}}\right)^{2} & \text { if } 0 \leq r \leq R \\
0 & \text { if } r<R\end{cases} \\
w_{\text {Green }} & =\frac{1}{2 \ell} \exp \left(-\frac{|r|}{\ell}\right),
\end{aligned}
$$

where, $r$ is the distance between the point of interest, $\underline{x}$, and the surrounding points, $\underline{\xi}$, and it is called the interaction radius. $\ell$ and $R$ are measures of the internal length scale. Jirásek and Bažant ${ }^{68}$ suggested that $\ell=R / \sqrt{7}$ in one dimension for the bell function. It should be noted that the choice of weighting function is to some degree arbitrary. The weight function needs to yield the local solution $\bar{f}=f$ when $f(\underline{x})$ is constant. Therefore $\int_{V} w(\xi) \mathrm{d} \xi=V$. It can also be noted that when the weighting function equals the Dirac delta function, the local solution is obtained.

While nonlocal theories provide a theoretically sound way to incorporate a length scale into the equations governing the material response, the practical implementation into FE codes is quite cumbersome. Standard FE programs only require information at integration points or nodes. Further, information is only needed one element at a time. In the case of nonlocal theories, information from further away is needed and information from neighboring elements is required. Secondly, deriving and including boundary conditions is not a trivial task either. ${ }^{72,73}$ It is therefore desirable to find a representation of the governing equations that is related to the nonlocal continuum theory, but where variables in the finite element formulation and assembly can be done one element at a time and where boundary conditions can be applied in the classical way. To that end, expand the field of some strain measure $\eta$ spatially through a Taylor series, ${ }^{74-76}$

$$
\eta(\underline{x}+\underline{\xi})=\eta(\underline{x})+\nabla \eta(\underline{x}) \underline{\xi}+\frac{1}{2 !} \nabla^{(2)} \eta(\underline{x}) \underline{\xi} \otimes \underline{\xi}+\frac{1}{3 !} \nabla^{(3)} \eta(\underline{x}) \underline{\xi} \otimes \underline{\xi} \otimes \underline{\xi}+\cdots+\frac{1}{n !} \nabla^{(n)} \eta(\underline{x}) \underline{\xi}^{(n)}
$$




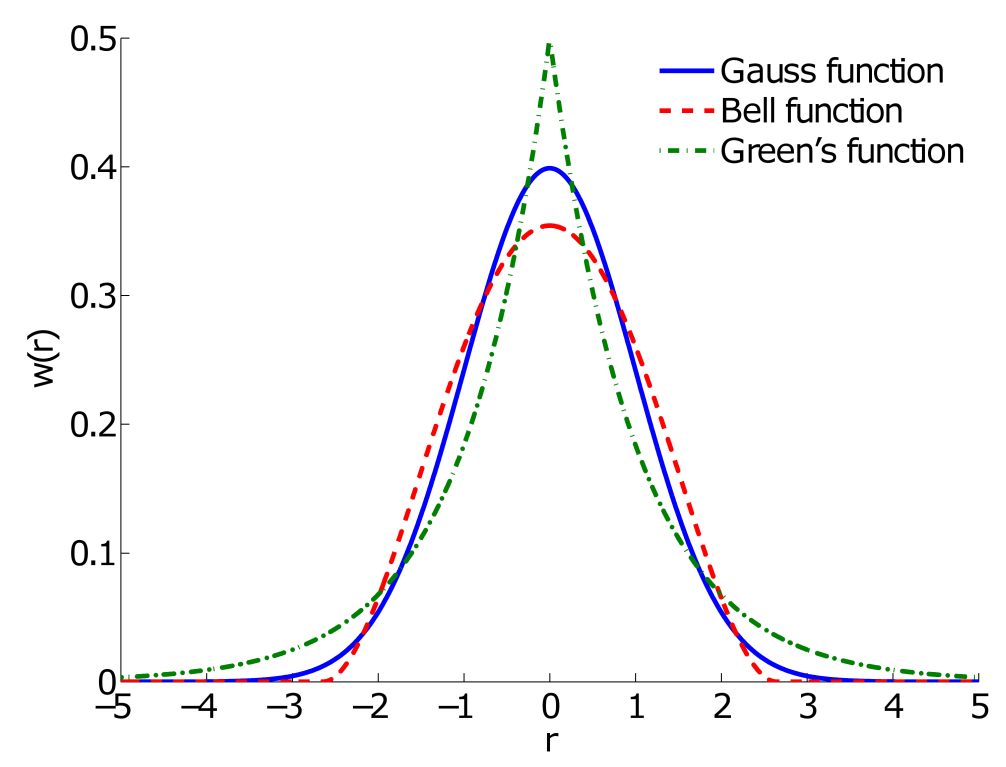

Figure 8. 1D normalized nonlocal weight functions; $\int_{-\infty}^{\infty} w(r) \mathrm{d} r=1$

with $\nabla^{n}$ denoting the $n$th order gradient operator and $\underline{\xi}^{(n)}$ is a dyadic product of $\mathrm{n}$ terms. Substituting this into the definition of a nonlocal field (33) yields

$$
\bar{\eta}(\underline{x})=\eta(\underline{x})+c_{1}^{2} \nabla^{2} \eta(\underline{x})+c_{2}^{4} \nabla^{4} \eta+\cdots
$$

$c_{1}$ and $c_{2}$ are expansion parameters. They have the dimension of length. Therefore in (37), a length scale parameter is present. By retaining only low order terms, one obtains the simplest approximation to nonlocal theory as,

$$
\bar{\eta}(\underline{x})=\eta(\underline{x})+c_{1}^{2} \nabla^{2} \eta(\underline{x})
$$

Due to the symmetry of the weighting function only even terms are retained, ${ }^{77}$ leading to an explicit second gradient formulation. The nonlocal variable depends explicitly on its local counterpart, which makes the evaluation especially easy. However this formulation is less suited for finite element implementation, because it requires $\mathcal{C}^{1}$-continuity in the nonlocal variable. That means, that at the finite element nodes, the variable and its derivative need to be continuous. This is necessary to ensure that the second derivative in equation (39) is evaluated with a non-trivial value. This type of formulation is well known from the FE solutions of Euler-Bernoulli beam equations, where displacements and their derivatives have to be continuous. The explicit gradient formulation has been used by Askes and co-workers ${ }^{78,79}$ to calculate the solution to gradient enhanced damage mechanics in a bar. However their numerical implementation used element free Galerkin solutions that has no requirements on continuity. Dorgan and Voyiadjis ${ }^{80}$ used explicit gradients and a mixed finite element formulation of the evolution of damage, and isotropic and kinematic plasticity to calculate the formation of shear bands. It should also be noted that the explicit gradient formulation is only weakly nonlocal. That is, the nonlocality is limited to an infinitesimal volume. From a finite element point of view this means that information of the nonlocal field is only important with respect to the neighboring elements and the exchange of that information is ensured by the $\mathcal{C}^{1}$-continuity requirement. Values from elements further away are not important. To remove these restrictions, implicit nonlocal formulations can be adopted. ${ }^{71,76,78,79,81,82}$ To that end, apply the second order gradient operator to equation (39). Then,

$$
\begin{aligned}
\nabla^{2} \bar{\eta} & =\nabla^{2} \eta+c_{1}^{2} \nabla^{4} \eta \\
\nabla^{2} \eta & =\nabla^{2} \bar{\eta}-c_{1}^{2} \nabla^{4} \eta
\end{aligned}
$$

and insert the result into the original equation (39). Then, 


$$
\bar{\eta}=\eta+c_{1}^{2}\left(\nabla^{2} \bar{\eta}-c_{1}^{2} \nabla^{4} \eta\right)
$$

Rename the constant $c_{1}$ to $\ell$ to signify its meaning as an internal length parameter, rearrange and neglect higher order terms to finally find,

$$
\bar{\eta}(\underline{x})-\ell^{2} \nabla^{2} \bar{\eta}(\underline{x})=\eta(\underline{x})
$$

This is termed an implicit formulation, because the nonlocal field cannot be written anymore directly but is instead implicitly stated through a differential relationship with the local field variable. While equation (39) is weakly nonlocal, equation (43) is strongly nonlocal. The nonlocal field variable now depends on a finite neighborhood around it. This can also been seen from a finite element point of view: like all elliptic partial differential equations that are solved with an implicit FE scheme, the entire body has to be considered at once. That is, all degrees of freedom of the FE formulation are solved for simultaneously. In the same manner it is necessary to consider the entire local field in the body to find the nonlocal field. Peerlings et al. ${ }^{71}$ have shown that implicit gradient models provide physically meaningful solutions when analyzing wave propagation. The wave speed in a bar was bounded for nonlocal and implicit gradient models, whereas that was not the case for an explicit nonlocal model. Note that during the derivation of the implicit gradient formulation, the assumption was made that the characteristic length parameter $\ell$ is small. However, Peerlings et al. ${ }^{71}$ have shown that the solution to equation (43) is identical to a nonlocal model when Green's function has been used as the weighting function. In that case there is no restriction on the length parameter $\ell$. Physically a larger length parameter increases the volume in which damage will occur. It will smooth out the transition from undamaged to damaged material because a higher weight is given to the derivatives.

Having obtained the definition of the nonlocal field variable, it is possible to restate the stress-strain relationship of the damaging law in equation (32), as,

$$
\underline{\underline{\sigma}}(\underline{x})=(1-D(\bar{\eta}(\underline{x}))) \underline{\underline{\underline{\underline{C}}}:} \underline{\underline{\varepsilon}}(\underline{x})
$$

The damage is now a function of the nonlocal strain measure, $\bar{\eta}$, which may be either obtained through the explicit or the implicit gradient formulation. As opposed to the damage, stress and strain are treated as local variables. Pijaudier-Cabot and Bažant ${ }^{73}$ commented on this by stating that without any damage present, the classical solution of elasticity should be obtained. A similar formulation has been used by Challamel. ${ }^{83}$ However he used the nonlocal damage field $\bar{D}$ directly instead of evaluating the nonlocal strain measure and then evaluating the damage locally as $D(\bar{\eta})$.

\section{Extension of nonlocal continuum damage model to cure of epoxy}

In the following the ideas of continuum damage will be combined with the ideas of the network creation during cure. Previously, every network was treated as a linear isotropic solid with unique elastic properties, cure shrinkage, thermal expansion coefficient and stress free reference configuration. Now each network will also have some damage $D$ associated with it. Specifically, recall the stress-strain relation from equation (24) and allow all networks to damage individually by pre-multiplying the per-network bulk modulus $K(s)$ and the per-network shear modulus $\mu(s)$ with a damage term of the form $(1-D(t, s))$.

$$
\begin{aligned}
\underline{\underline{\sigma}}(t)= & \int_{0}^{t} \dot{\phi}(s)(1-D(t, s))\left[K(s) \operatorname{tr}\left(\underline{\underline{\varepsilon}}(t)-\underline{\underline{\varepsilon}}(s)+\underline{\underline{\varepsilon_{c}}}(s)-\underline{\underline{1}} \alpha(s) \Delta T(t, s)\right)\right. \\
& \left.+2 \mu(s)\left(\underline{\underline{\varepsilon}}(t)-\underline{\underline{\varepsilon}}(s)+\underline{\underline{\varepsilon_{c}}}(s)-\underline{\underline{1}} \frac{1}{3} \operatorname{tr}\left(\underline{\underline{\varepsilon}}(t)-\underline{\underline{\varepsilon}}(s)+\underline{\underline{\varepsilon_{c}}}(s)\right)\right)\right] \\
& +(1-\phi(t)) K_{l i q} \operatorname{tr}\left(\underline{\underline{\varepsilon}}(t)-\underline{\underline{1}} \alpha_{l i q} \Delta T(t)\right) \underline{\underline{1}}
\end{aligned}
$$

The damage parameter $D(t, s)$ depends on the current state at time $t$ as well as on the history at times $s$. Specifically it depends on the nonlocal field variable $\bar{\eta}(t, s)$. The nonlocal field variable depends on the local field variable through equation (43). That is,

$$
\bar{\eta}(t, s)-\ell^{2} \nabla^{2} \bar{\eta}(t, s)=\eta(t, s)
$$


The nonlocal field variables at different times in history are independent of each other. Each network created at time $s=t_{i}$, is independent from the network created at time $s=t_{j}$. That means equation (46) has to be solved for all histories $0 \leq s \leq t$, leading to an infinite number of equations. At this point another problem regarding the numerical formulation of equation (45) should be mentioned: taking the integral with respect to time becomes increasingly difficult. In the absence of damage, it is possible to split up the integral into different terms and evaluate it using a running update. That means the integral could be divided into parts only depending on the current state at time $t$ and other parts that depend only on the history at times $s$. This is not possible when damage is included. The dependence of damage on the current state as well as the history makes it necessary to save the histories of stress, strain, cure, curing rate, temperature and damage during a FE simulation. It should be noticed, that the integral only needs to be evaluated during a finite time interval, $0<t<t_{\text {cure }}$. At the curing time, $t_{\text {cure }}$, the curing process is regarded to be complete. Mathematically, full cure is approached asymptotically. Therefore $\phi_{\text {cure }}=95 \%$ has been chosen for convenience such that $t_{\text {cure }}=t\left(\phi=\phi_{\text {cure }}\right)$. At full cure the rate of cure is zero and the portion of the integral relating to subsequent times will be zero, as well. It is now possible to numerically evaluate the integral using for example the trapezoidal or Simpson's rule. Here, the kernel of the integral only needs to be known at selected time intervals $0=t_{0}<t_{1}<t_{2}<\cdots<t_{N}=t_{\text {cure }}$. The amount of history information saved needs to be stated prior to performing a FE simulation. On the other hand it is not known a priori how and when full cure will be achieved. Therefore the curing process is divided into equal intervals with respect to degree of cure. That is, if $N$ time slots are allotted to save history information, the times are given by $t_{0}=t(\phi=0)=0, t_{1} \geq t\left(\phi=\phi_{\text {cure }} /(1-N)\right), \ldots, t_{N-1} \geq t_{\text {cure }}$. The time steps are chosen by the finite element program and generally do not coincide with the times when the prescribed curing stages are reached. Therefore, the times when to save the history information do not usually follow entirely the desired even spacing in degree of cure. Inherent to this proposed integration scheme is the assumption that during the curing, the loading and boundary conditions are sufficiently smooth and not subject to oscillations, which is true for any realistic curing and loading scenario.

Finally, the nonlocal field variable only needs to be determined at the times that are used to numerically evaluate the integral. That is,

$$
\begin{aligned}
\bar{\eta}\left(t, t_{0}\right)-\ell^{2} \nabla^{2} \bar{\eta}\left(t, t_{0}\right) & =\eta\left(t, t_{0}\right) \\
\bar{\eta}\left(t, t_{1}\right)-\ell^{2} \nabla^{2} \bar{\eta}\left(t, t_{1}\right) & =\eta\left(t, t_{1}\right) \\
& \vdots \\
\bar{\eta}\left(t, t_{N-1}\right)-\ell^{2} \nabla^{2} \bar{\eta}\left(t, t_{N-1}\right) & =\eta\left(t, t_{N-1}\right)
\end{aligned}
$$

In the finite element program, the nodal variables will be grouped by times. That is, $\bar{\eta}=\left[\bar{\eta}\left(t_{0}\right)\right.$, $\left.\bar{\eta}\left(t_{1}\right), \ldots, \underline{\bar{\eta}}\left(t_{N-1}\right)\right]^{\mathrm{T}}$, where $\overline{\bar{\eta}}\left(t_{i}\right)$ are the nodal values of the nonlocal field variable belonging to the network formed at time $t_{i}$. The damage should only depend on the mechanical strain in a network. Therefore the (local) field variable $\eta(t, s)$ needs to be some invariant of the mechanical strain $\underline{\varepsilon}^{m}(t, s)$. The two most promising choices are the maximum principal mechanical strain, which will be used in the following, or the von Mises mechanical strain (the deviatoric part of the strain tensor). Both, von Mises strain and maximum principal strain, collapse to the uniaxial strain in a one dimensional tension problem when only end loads or displacements are prescribed. Other invariants of the strain might be possible to be used, for example the mean hydrostatic strain.

The mechanical strain can be written as,

$$
\underline{\underline{\varepsilon}}^{m}(t, s)=\underline{\underline{\varepsilon}}(t)-\underline{\underline{\varepsilon}}(s)+\underline{\underline{\varepsilon}}_{c}(s)-\alpha(s)(T(t)-T(s))
$$

where, $\varepsilon(t)$ is the total strain at current time, $t, \varepsilon(s)$ is the total strain at the time of the creation of the network $, s, \varepsilon$ is the cure shrinkage of the network created at time $s, \alpha(s)$ is the coefficient of thermal expansion and $\bar{T}(t)$ and $T(s)$ are the current temperature and the temperature at the time the network was created.

\section{Determination of material properties}

Several material properties of the epoxy need to be determined in order to use the described model in finite element calculation. The properties include elastic, thermal, chemical, and damage parameters. 


\section{A. Elastic properties}

The plane wave modulus, shear modulus and degree of cure are measured as a function of time through concurrent Raman (RLS) and Brillouin (BLS) light scattering. ${ }^{84-86}$ The plane wave modulus $M$ is the elastic constant measured in a uniaxial strain field. It is related to the Lamé constants and the bulk modulus by $M=\lambda+2 \mu=K+\frac{4}{3} \mu$. Raman scattering provides cure information and Brillouin light scattering provides mechanical information. This optical measurement method has the advantage of yielding mechanical and chemical properties non-destructively. In contrast, classical methods that measure the mechanical properties during cure, such as rheometers or bending tests, ${ }^{87}$ actively influence the boundary value problem that is needed to extract the per-network properties for this approach.

It is important to note that experimentally, the response of all networks is measured simultaneously. The individual response of each network has to be adjusted so that the total simulated response follows the total experimentally measured response. To this end, assume that the network is cured in a uniaxial strain field. After some time, a sudden jump in strain is applied. The jump needs to be considered for all times during the curing history, because the material properties need to be extracted for all times during the curing history. The stress response is measured in the direction the strain jump is applied, and in the perpendicular direction. The boundary value problem (BVP) is depicted in figure 9. It also shows the meaning of the plane wave modulus, $M$. Two coordinate systems are shown, one aligned with the strain direction and an internal coordinate system with some arbitrary direction. The latter is a useful tool to ensure the numerical implementation into the finite element program is correct. While reaction forces are reported in the global coordinate system lined up with the axis of deformation, internally, the finite element program calculates stresses and strains with respect to the rotated coordinate system. The stress and strain tensor will be fully populated and possible error in the programming of the material model will be easier to find.

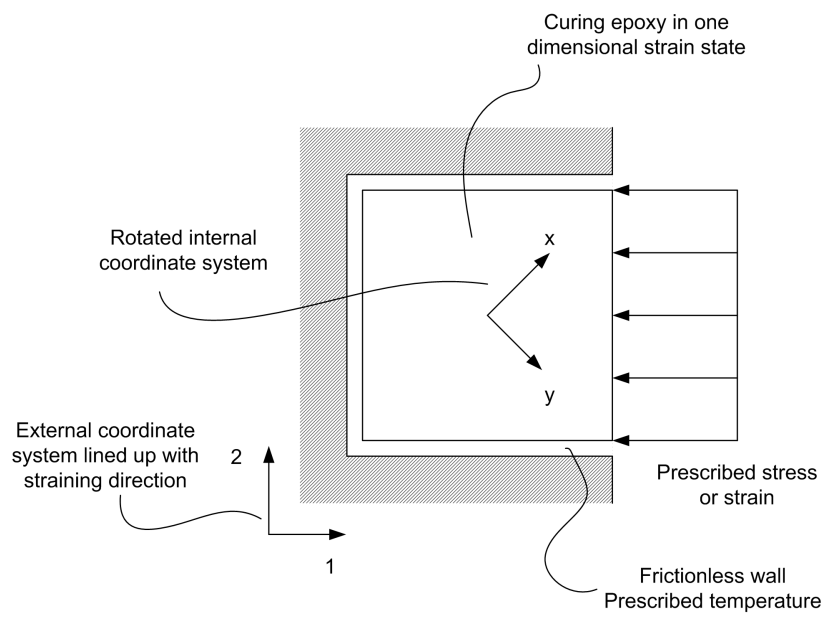

Figure 9. Uniaxial strain boundary value problem used to determine material properties

Ignoring the influence of temperature, the stresses before and after the jump, respectively, are:

$$
\begin{gathered}
\underline{\underline{\sigma}}=\left[\begin{array}{ccc}
\sigma_{11} & 0 & 0 \\
0 & \sigma_{22} & 0 \\
0 & 0 & \sigma_{33}=\sigma_{22}
\end{array}\right] \\
\underline{\underline{\sigma}}+\Delta \underline{\underline{\sigma}}=\left[\begin{array}{ccc}
\sigma_{11}+\Delta \sigma_{11} & 0 & 0 \\
0 & \sigma_{22}+\Delta \sigma_{22} & 0 \\
0 & 0 & \sigma_{33}=\sigma_{22}+\Delta \sigma_{22}
\end{array}\right]
\end{gathered}
$$

Thus,

$$
\sigma_{11}=\int_{0}^{t} \dot{\phi}\left\{K\left[\varepsilon(t)-\varepsilon(s)+3 \varepsilon_{c}(s)\right]+\frac{4}{3} \mu[\varepsilon(t)-\varepsilon(s)]\right\} \mathrm{d} s+(1-\phi(t)) K_{l i q} \varepsilon(t)
$$




$$
\begin{aligned}
\sigma_{11}+\Delta \sigma_{11}=\int_{0}^{t} \dot{\phi}\left\{K\left[\varepsilon(t)+\Delta \varepsilon-\varepsilon(s)+3 \varepsilon_{c}(s)\right]+\right. & \left.\frac{4}{3} \mu[\varepsilon(t)+\Delta \varepsilon-\varepsilon(s)]\right\} \mathrm{d} s \\
& +(1-\phi(t)) K_{l i q}(\varepsilon(t)+\Delta \varepsilon)
\end{aligned}
$$

The difference between the two is

$$
\begin{aligned}
\Delta \sigma_{11} & =\Delta \varepsilon \int_{0}^{t} \dot{\phi}\left\{K(s)+\frac{4}{3} \mu(s)\right\} \mathrm{d} s+\Delta \varepsilon(1-\phi) K_{l i q} \\
& =\Delta \varepsilon \int_{0}^{t} \dot{\phi} M(s) \mathrm{d} s+\Delta \varepsilon(1-\phi) K_{l i q}
\end{aligned}
$$

The strain tensor of the uniaxial strain field is:

$$
\underline{\underline{\varepsilon}}=\left[\begin{array}{ccc}
\varepsilon & 0 & 0 \\
0 & 0 & 0 \\
0 & 0 & 0
\end{array}\right]
$$

This can be written as

$$
\Delta \sigma_{11}=\Delta \varepsilon_{11} M_{t o t}(t)
$$

where

$$
\begin{aligned}
M_{t o t} & =\int_{0}^{t} \dot{\phi} M(s) \mathrm{d} s+(1-\phi(t)) K_{l i q} \\
& =\int_{0}^{t} \dot{\phi}\left\{K(s)+\frac{4}{3} \mu(s)\right\} \mathrm{d} s+(1-\phi(t)) K_{l i q}
\end{aligned}
$$

is the total plane wave modulus, and $M(s)$ is the plane wave modulus of each network formed at time $s$. Differentiating with respect to time, $t$, re-arranging and applying the chain rule yields the per-network properties.

$$
M(\phi)=\frac{\mathrm{d} M_{t o t}(\phi)}{\mathrm{d} \phi}+K_{l i q}
$$

The same BVP can be used to determine a second set of properties. For the direction perpendicular to the one in which the strain is applied, it follows that:

$$
\begin{aligned}
\Delta \sigma_{22} & =\Delta \varepsilon \int_{0}^{t} \dot{\phi}\left(K(s)-\frac{2}{3} \mu(s)\right) \mathrm{d} s+\Delta \varepsilon(1-\phi) K_{l i q} \\
& =\Delta \varepsilon \int_{0}^{t} \dot{\phi} \lambda(s) \mathrm{d} s+\Delta \varepsilon(1-\phi) K_{l i q}
\end{aligned}
$$

For this particular BVP,

$$
\Delta \sigma_{22}=\Delta \varepsilon_{11} \lambda_{t o t}(t)
$$

where

$$
\lambda_{t o t}=\int_{0}^{t} \dot{\phi}(s) \lambda(s) \mathrm{d} s+(1-\phi(t)) K_{l i q}
$$

so that the per-network Lamé constant is 


$$
\lambda(\phi)=\frac{\mathrm{d} \lambda_{t o t}(\phi)}{\mathrm{d} \phi}+K_{l i q}
$$

This can be transformed to yield the per network shear and bulk moduli:

$$
\begin{aligned}
\mu(\phi) & =\frac{\mathrm{d} \mu_{t o t}}{\mathrm{~d} \phi} \\
K(\phi) & =\frac{\mathrm{d} K_{t o t}}{\mathrm{~d} \phi}+K_{l i q}
\end{aligned}
$$

Figures 10(a) and 10(b) show representative experimental data for $M$ and $\mu$ as functions of extent of cure. For mathematical convenience the transition of these material properties is currently modeled with an analytical function:

$$
\begin{aligned}
X_{\mathrm{tot}}(\phi) & =\frac{\arctan \left(\left(\phi-\frac{1}{2}\right) \beta_{X}\right)}{\arctan \left(\frac{\beta_{X}}{2}\right)}\left(X_{f}-X_{s}\right)+\left(\frac{X_{f}+X_{s}}{2}\right) \\
X & =\{\mu, M\}
\end{aligned}
$$

This leads to closed form solutions for the per-network properties:

$$
\begin{aligned}
\mu(\phi) & =\frac{1}{2} \frac{\beta_{\mu}\left(\mu_{f}-\mu_{s}\right)}{\left(1+(\phi-1 / 2)^{2} \beta_{\mu}^{2}\right) \arctan \left(1 / 2 \beta_{\mu}\right)} \\
M(\phi) & =\frac{1}{2} \frac{\beta_{M}\left(M_{f}-M_{s}\right)}{\left(1+(\phi-1 / 2)^{2} \beta_{M}^{2}\right) \arctan \left(1 / 2 \beta_{M}\right)}+K_{l i q} \\
K & =M-\frac{4}{3} \mu
\end{aligned}
$$

It should be noted that in reality, gelation occurs. Until gelation, the shear modulus of the material is undetectable and the resin behaves like a viscous fluid. This is shown in figure 10(b). In the current model, a small amount of shear stiffness is assumed even for low amounts of curing. Finally, the bulk modulus of the liquid epoxy is the one measured at the beginning of the experiment.

$$
K_{l i q}=K_{t o t}(0)
$$

\section{B. Cure shrinkage}

To determine the correct cure shrinkage a boundary value problem needs to be found, where the change in volume can be measured. Therefore, consider again the uniaxial strain BVP of a curing epoxy sample as shown in figure 9. The specimen is free to expand or contract in the 1-direction. No stresses are applied in this direction. The stress and strain tenors are,

$$
\underline{\underline{\sigma}}=\left[\begin{array}{ccc}
\sigma_{11}=0 & 0 & 0 \\
0 & \sigma_{22} & 0 \\
0 & 0 & \sigma_{33}=\sigma_{22}
\end{array}\right]
$$

and

$$
\underline{\underline{\varepsilon}}=\left[\begin{array}{lll}
\varepsilon & 0 & 0 \\
0 & 0 & 0 \\
0 & 0 & 0
\end{array}\right]
$$

The stress in the 1-direction is given by 


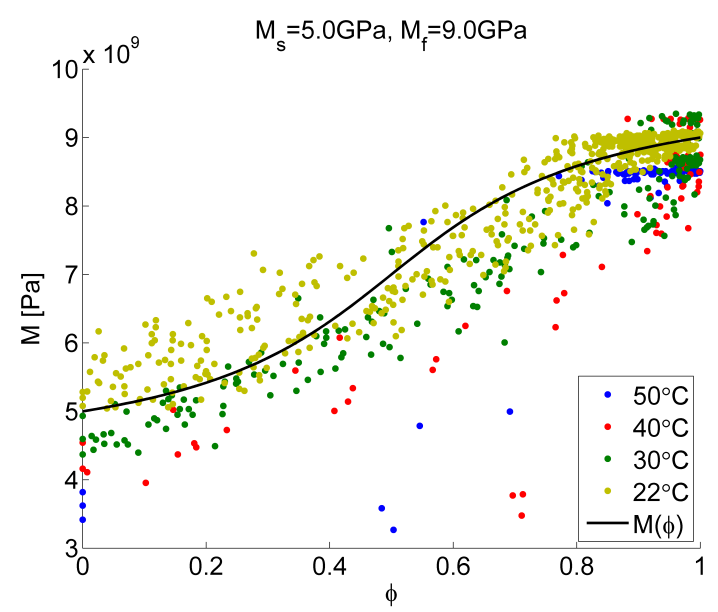

(a) Plane wave modulus

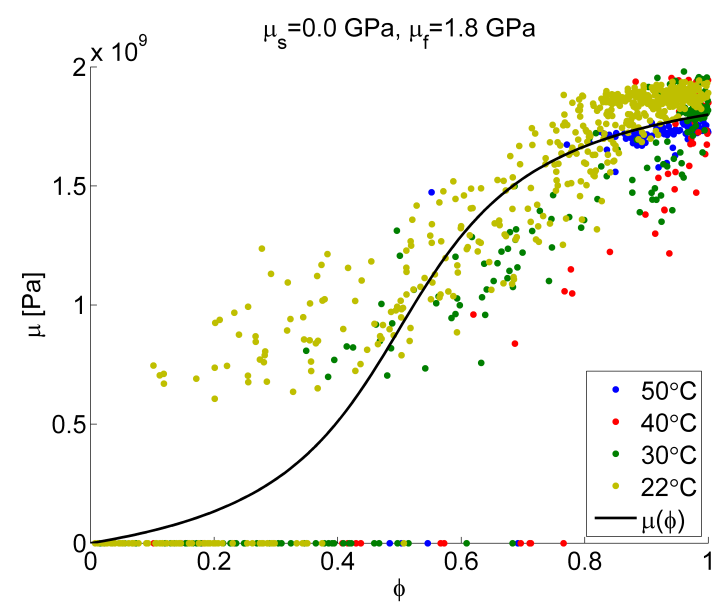

(b) Shear modulus

Figure 10. Experimental results of Brillouin and Raman light scattering

$$
\sigma_{11}=\int_{0}^{t} \dot{\phi}(s) M(s)(\varepsilon(t)-\varepsilon(s)) \mathrm{d} s+3 \int_{0}^{t} \varepsilon_{c}(s) K(s) \dot{\phi}(s) \mathrm{d} s+(1-\phi(t)) \varepsilon(t) K_{l i q},
$$

and it is equal to zero. There is a monotonic relation between time, $t$, and degree of cure, $\phi$. A change in variables of $t \rightarrow \Phi=\phi(t)=$ and $\dot{\phi} \mathrm{d} s \rightarrow \mathrm{d} \phi$ leads to:

$$
\sigma_{11}(\Phi)=\int_{0}^{\Phi} M(\phi)(\varepsilon(\Phi)-\varepsilon(\phi)) \mathrm{d} \phi+3 \int_{0}^{\Phi} \varepsilon_{c}(\phi) K(\phi) \mathrm{d} \phi+(1-\Phi) \varepsilon(\Phi) K_{l i q}=0
$$

Differentiating with respect to the upper limit, observing that no stress was applied in the 1-direction and rearranging, yields the final answer for the cure shrinkage

$$
\varepsilon_{c}(\Phi)=\frac{1}{3 K(\Phi)}\left[\left(\varepsilon(\Phi)-(1-\Phi) \frac{d \varepsilon(\Phi)}{\mathrm{d} \Phi}\right) K_{l i q}-\frac{d \varepsilon(\Phi)}{\mathrm{d} \Phi} \int_{0}^{\Phi} M(\phi) \mathrm{d} \phi\right]
$$

The total cure shrinkage of all networks $\varepsilon(\Phi)$ can be measured through a gravimetric test method. ${ }^{88}$ Table 1 gives an account of the results that have been obtained for various epoxy systems.

\begin{tabular}{|c|c|l|}
\hline Cure shrinkage/volume change & Material & Reference \\
\hline $6.9 \%$ & MY750 & 88 \\
$4.5 @ 100^{\circ} C, 200$ bar & DGEBA/MCDEA & 55 \\
$>1 \%$ & EPON828/DEP & 43 \\
$2.75 \%$ after gelation & Bisphenol F & 89 \\
$<2 \%$ & Epikote $828 /$ MNA/BDMA & 90 \\
$2 \%$ & current study & \\
\hline
\end{tabular}

Table 1. Cure shrinkage in various epoxy materials

\section{Thermal expansion and thermal conductivity}

Data for thermal expansion is sparse in the literature and given in table 2. Since no data was obtainable for non-crosslinked epoxy the thermal expansion coefficients are the same for cured and uncured networks. Thermal conductivity can be modeled through a rule of mixture relation.

$$
K=K_{l i q}(1-\phi)+K_{\text {all networks }}
$$




\begin{tabular}{|c|c|l|}
\hline $\begin{array}{c}\text { (Linear) Coefficient of } \\
\text { thermal expansion }(\mathrm{ppm} / \mathrm{K})\end{array}$ & Material & Reference \\
\hline 65 & Bisphenol F/HHPA & 91 \\
61 & Epon862/Epikure 3234 & 92 \\
\hline
\end{tabular}

Table 2. Thermal expansion coefficient of various epoxies

Varshney et al. ${ }^{93}$ have investigated the thermal conductivity of $\mathrm{EPON}^{\mathrm{TM}} 862$ resin, curing agent DETDA and their mixture as well as the cross linked epoxy using molecular dynamics simulations. However, the data differs more for different types of simulations than for crosslinked vs. non-crosslinked networks. Therefore, currently a constant value is chosen for the thermal conductivity.

\section{Damage properties}

In addition to the elastic properties as a function of cure which are obtained through BLS and RLS, properties relating the onset and evolution of damage response need to be found. Specifically, the characteristic length, $\ell$, the strain at which damage initiates, $\varepsilon_{f}$, and the ultimate strain, where full damage is reached, $\varepsilon_{u}$, are needed. Ideally, these values are determined for each network individually. To accomplish this, a corresponding experiment would need to be conducted at various stages of the curing process. Possible experiments include a double cantilever beam experiment as described below, or a compact tension test. For a first estimate of the damaging material properties, assume the following: no cure strain $\left(\underline{\underline{\varepsilon}}_{c}=0\right)$ and external strain are applied to the specimen during cure, the temperature is constant throughout and all networks experience the same damage behavior. Then the stress as given in equation (45) can be simplified to give,

$$
\underline{\underline{\sigma}}\left(t>t_{\text {cure }}\right)=(1-D(t))\left[\left(\underline{\underline{1}} \frac{1}{3} \operatorname{tr} \underline{\underline{\varepsilon}}(t)\right) \int_{0}^{t_{\text {cure }}} \dot{\phi} K(s) \mathrm{d} s+\left(\underline{\underline{\varepsilon}}(t)-\underline{\underline{1}} \frac{1}{3} \operatorname{tr} \underline{\underline{\varepsilon}}(t)\right) \int_{0}^{t_{\text {cure }}} \dot{\phi} 2 \mu(s) \mathrm{d} s\right]
$$

This means that the cured epoxy will be regarded as a classical isotropic solid, and equation (78) has now the same form as equation (32).

The remaining question is the proper choice of the length scale parameter, $\ell$, and the ultimate strain, $\varepsilon_{u}$. $\ell$, has influence on the size of the zone or volume in which damage occurs. The shape of the stress-strain curve, and thus the value of $\varepsilon_{u}$ prescribes how much energy is dissipated per unit volume during the damage process. The length scale parameter $\ell$ is chosen to be $0.1 \mathrm{~mm}$. To find a corresponding ultimate strain value, an estimate of energy that is related to the damage process is needed. This has been investigated before, on the laminate level, using Shapery theory. ${ }^{94-96}$ For pure epoxy such values are not readily available. However, fracture energy is an energetic value related to failure that has been thoroughly studied. ${ }^{68}$ It will therefore be used to obtain a value of $\varepsilon_{u}$. Using the data for EPON ${ }^{\mathrm{TM}} 862$ resin with EPIKURE ${ }^{\mathrm{TM}} 3234$ curing agent from the literature, as given in table 3, the consistent damage related properties, as shown in Table 4 were determined using simulation of a double cantilever cantilever beam test. It should be noted that when applying an idealized stress-strain curve, as shown in figure 7 , the yield strain calculated from the manufacturer data presented is $2.55 \%$. This value was used for the failure onset strain, $\varepsilon_{f}$.

\section{Cure and subsequent mechanical response of a woven textile composite unit cell}

\section{A. Problem description}

The material model has been programmed into an ABAQUS user element ${ }^{99}$ and a finite element simulation has been performed of the curing of a $1 \times 1$ plain weave representative unit cell (RUC). This is one of the simplest textile architectures and it allows for a creation of the problem geometry. In the previous studies, the tows which consist of matrix and fibers have been homogenized, so that a unit cell consisting of a few homogenized tows and the matrix material in between ${ }^{2,5}$ have been utilized for model development. For these models, a viable homogenization technique needs to exist. Due to the novel nature of the proposed 


\begin{tabular}{|cccl|}
\hline Property & Value & Unit & Reference \\
\hline Modulus & 3.24 & $\mathrm{GPa}$ & 92 \\
& 2.76 & $\mathrm{GPa}$ & 97 \\
Yield Strength & 82.7 & $\mathrm{MPa}$ & 92 \\
& 86 & $\mathrm{MPa}$ & 97 \\
Yield strain & 6.6 & $\%$ & 92 \\
Fracture energy & 606 & $\mathrm{~J} / \mathrm{m}^{2}$ & 97 \\
& 280 & $\mathrm{~J} / \mathrm{m}^{2}$ & 98 \\
\hline
\end{tabular}

Table 3. Properties of EPON 862, obtained from literature

\begin{tabular}{|ccc|}
\hline Property & Value & Unit \\
\hline Final elastic modulus $E$ & 4.95 & GPa \\
Damage initiation strain $\varepsilon_{f}$ & 2.55 & $\%$ \\
Ultimate strain $\varepsilon_{u}$ & 5.0 & $\%$ \\
Length parameter $\ell$ & 0.1 & $\mathrm{~mm}$ \\
\hline
\end{tabular}

Table 4. Damage related properties of EPON 862 used in simulations

curing model such a homogenization technique is not readily available. An exception is of course the use of a fine scale finite element model within a coarse scale finite element model. However, currently such an approach would be computationally very expensive. As an alternative to homogenization of the tows, they will be modeled using micromechanics. That is, the tows will be represented as a collection of a few discrete fibers and matrix. The size of the fibers is chosen such that the volume fraction and the outer dimension of the tow is equivalent to the dimensions of an actual tow. In previous studies it was shown that for a particular class of material properties the response of a unit cell consisting of 20 fibers or more is insensitive to the packing. ${ }^{100}$ A similar behavior is expected when the fibers are arranged in undulating tows, instead of uniaxial RUCs. However, in order to keep the computational size manageable, each tow will consist of 7 fibers, which are arranged in a compressed hexagon, as shown in figure 11(c). The RUC is shown without fibers in figure 11(a) and with fibers and matrix in figure 11(b)..

First, the RUC is fully cured using the formulation and implementation described earlier. Next, a uniaxial displacement controlled tension test is performed. To allow for the tension test subsequent to cure, boundary conditions as depicted in figure 12 are needed. The surfaces in the 2-direction and 3-direction are unconstrained and traction free at all times. The two surfaces with a normal along the 1-direction are also free to move in the 2-direction and 3-direction. However, one of the two surfaces is completely constrained in the 1-direction. The other surface with a normal in the 1-direction is constrained such that plane sections remain plane. During cure that plane is free to expand and contract. During loading a concentrated force is applied to a reference node, which in turn is coupled to that surface. These constraints are necessary, because during cure the RUC deforms due to shrinkage and thermal effects, and boundary conditions are always applied with respect to the undeformed shape, which is initially flat.

\section{B. Results and discussion}

Figure 14 shows the normalized results of a cure and tension simulation. The displacement applied at one side of the unit cell has been normalized by the RUC length to give an average strain. The total reaction force has been normalized by the cross-sectional area to give an average stress. Five different cases have been considered in figure 14. They are summarized in table 5 . In Case 1 , the RUC has been cured at $50^{\circ} \mathrm{C}$ for one hour and was then cooled back down to room temperature. The inelastic material properties used are shown in table 4 and the evolution of elastic properties is given in equations (68) and (70). Case 2 is identical to Case 1, except that the global cure shrinkage has been increased from $2 \%$ to $6 \%$. This cure shrinkage is comparable to the one seen in HETRON ${ }^{\mathrm{TM}}$ resin, which has been used as a matrix material in previous studies. ${ }^{1-5}$ In Case 3, neither cure shrinkage nor thermal expansion occurs, which is equivalent to $\alpha(s)=0$ and $\varepsilon_{c}=0$. Therefore the fiber and matrix are stress free prior to mechanical loading. In Case 4, 


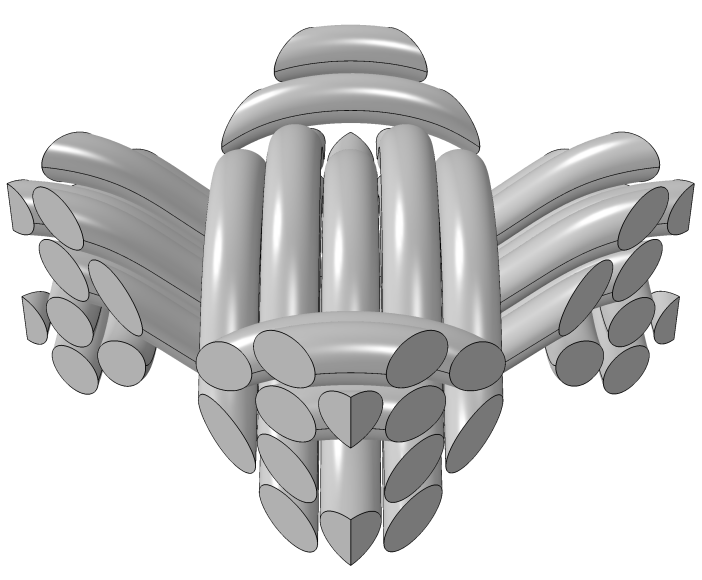

(a) Only fibers shown

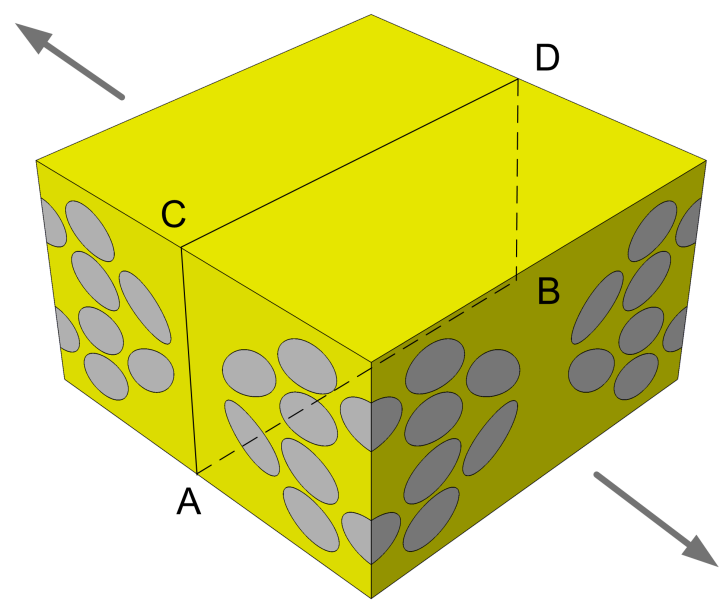

(b) Fibers and matrix show, loading direction indicated

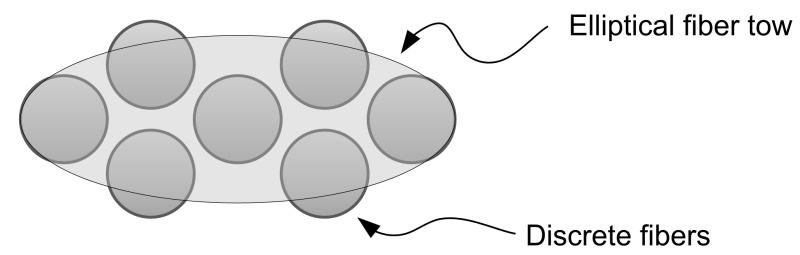

(c) Cross-section of idealized tow

Figure 11. 1x1 plain weave RUC

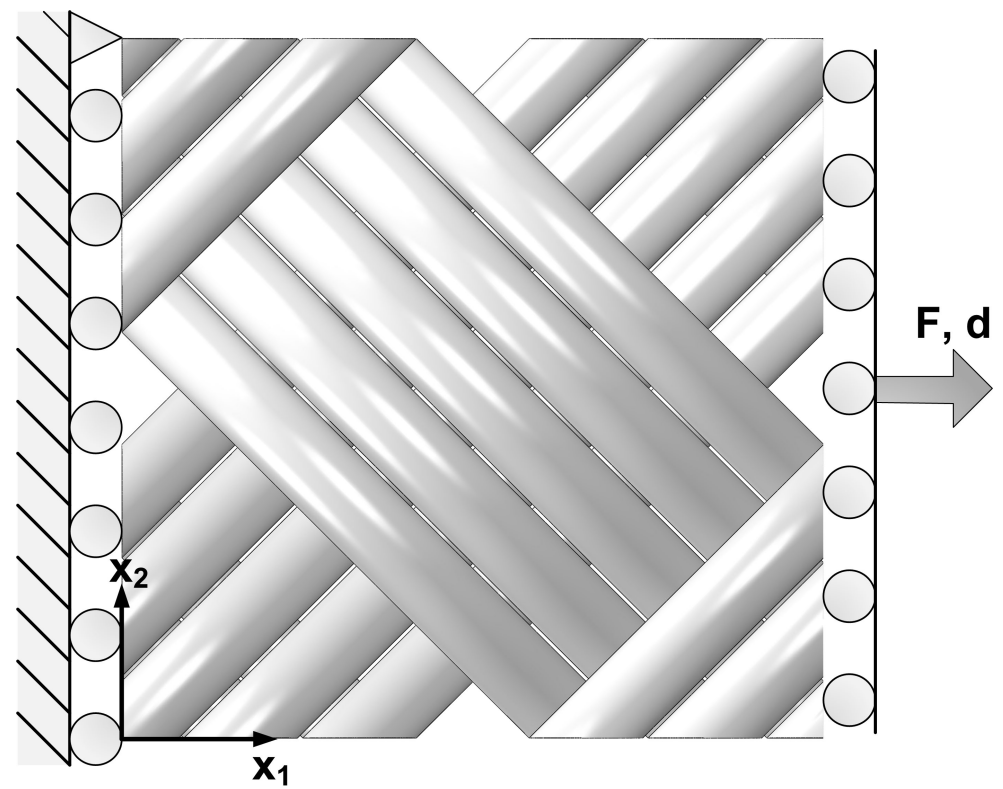

Figure 12. Top view of RUC to show boundary conditions applied during and after cure (only fibers are shown) 
only cure shrinkage is considered, which is a material state that can be obtained by curing and loading at the same temperature. Finally, Case 5 does not allow for any damage to occur during the cure and also during subsequent mechanical loading. In this case the loading response is entirely linear.

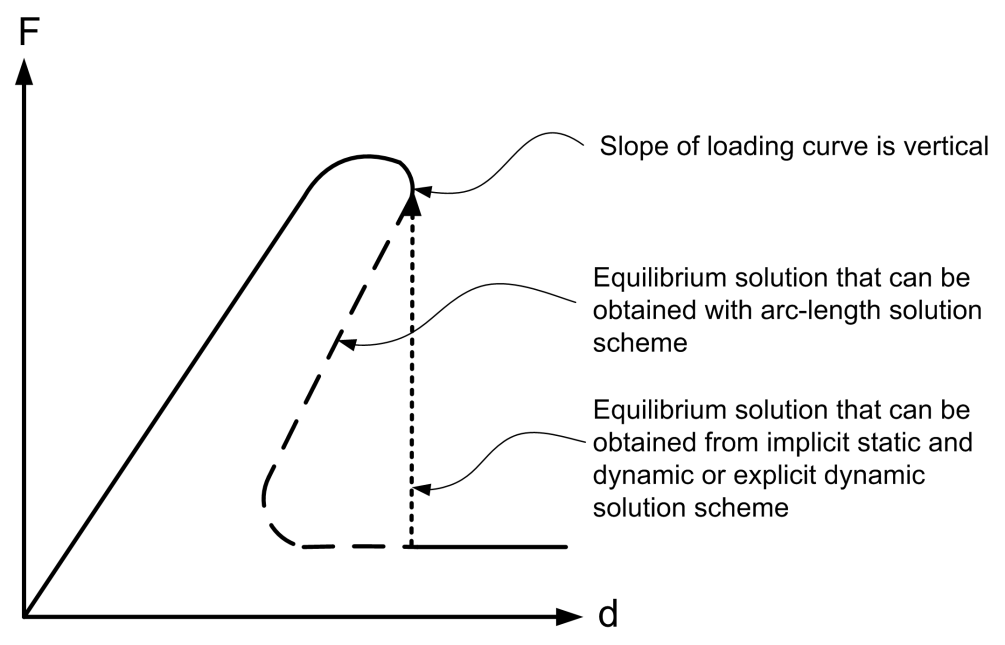

Figure 13. Snap-back and snap-through in damaging models

\begin{tabular}{|c|c|c|c|c|}
\hline Case & $\begin{array}{c}\text { Cure } \\
\text { shrinkage }\end{array}$ & $\begin{array}{l}\Delta T \text { at the } \\
\text { end curing step }\end{array}$ & $\begin{array}{l}\text { Damage } \\
\text { included }\end{array}$ & Comments \\
\hline 1 & $2 \%$ & $30^{\circ} \mathrm{C}$ & yes & EPON 862 Baseline \\
\hline 2 & $6 \%$ & $30^{\circ} \mathrm{C}$ & yes & $\begin{array}{l}\text { This case corresponds to an } \\
\text { elevated cure shrinkage that } \\
\text { is found in HETRON }{ }^{\mathrm{TM}} \text { resin }\end{array}$ \\
\hline 3 & $0 \%$ & $0^{\circ} \mathrm{C}$ & yes & Room temperature cure \\
\hline 4 & $2 \%$ & $0^{\circ} \mathrm{C}$ & yes & Room temperature cure \\
\hline 5 & $2 \%$ & $30^{\circ} \mathrm{C}$ & no & Linear properties and response \\
\hline
\end{tabular}

Table 5. The 5 cases simulated for a woven RUC with carbon fibers and EPON 862 resin

In Cases 1-4, a large linear regime was observed and followed by a small non-linear regime. The lack of prolonged softening behavior can be explained with the help of figure 13. It has been seen in the literature $^{76,101}$ that upon tensile loading a snap-back of the load deflection curve can be observed. That means that with continued softening due to accumulated damage, the load and displacement have to be lowered in subsequent iterations to find an equilibrium position. To successfully calculate such a snap back behavior, an arc-length method needs to employed, such as the RIKS method. ${ }^{102,103}$ If a standard implicit finite element solution scheme is used (without an arc-length solver), it is not possible to predict such a snap-back behavior. Instead a snap-trough type behavior, when the loading slope becomes vertical leads to solution divergence. At this point, a drop in load is observed and the subsequent equilibrium solution cannot be found by a standard implicit finite element solver. If the solver is an explicit solver, than a solution can be found, but there is additional kinetic energy, that enters the problem. In the current work, which models the evolution of material properties with time, the use of the RIKS method to solve the equilibrium equations is not straight forward, because time is not an explicit input to such a solution scheme.

Figure 14 shows the macroscopic stress, $\sigma$, and the macroscopic strain $E$ for the 5 cases that were studies. The maximum stresses, $\Sigma$, in Case 1, 3 and 4 are similar. The maximum $\Sigma$ is largest in Case 3, where no built-in stresses due to cure are present. The next largest maximum $\Sigma$ occurs in Case 4, where only internal stresses due to chemical shrinkage were considered, but not thermal gradients in time because the curing and loading were performed at the same temperature. Case 1 contains stresses before mechanical loading due to both chemical shrinkage and temperature changes in time. The maximum $\Sigma$ is therefore lower. However, in Case 1,2 and 4 no damage was observed at the end of the curing cycle. Case 2 is different in that regard. Here 
damage occurs already during cure and the matrix material starts to soften locally prior to the application of mechanical load. This is shown in figure 16(c). This RUC also shows a significantly reduced maximum $\Sigma$ in figure 14. While the maximum $\Sigma$ for Case 3 is $142 \mathrm{MPa}$, the maximum for Case 2 is $94 \mathrm{MPa}$, which is an effective reduction of over $30 \%$ of the maximum $\Sigma$.

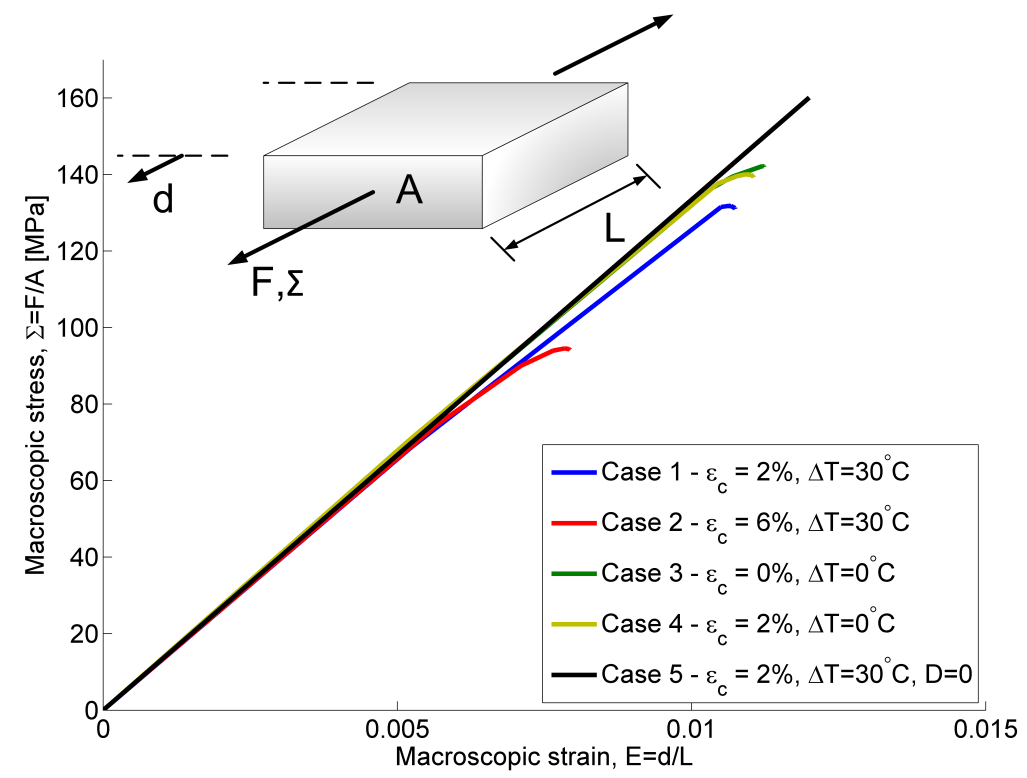

Figure 14. Global deformation response of woven RUC

To further investigate the reduction in maximum $\Sigma$, the internal stress and damage states of Case 1 and Case 2 at the end of the curing process and shortly before reaching the maximum macroscopic strain, $E$, due to mechanical loading are shown in figures 15 and 16. Figure 15(a) shows the internal maximum principal stresses, $\sigma_{\max , 1}$, in Case 1 due to cure, before external loads are applied. The maximum stress is largest in the center of the fiber tow and is smallest away from the fibers. In the center of the fiber tow, the fibers act as a constraints that prevent the matrix from shrinking as cure progresses and therefore stresses build up in that region. From figure 16(a) it can be seen that no damage is present before external mechanical loading is applied. Figure 15(b) shows $\sigma_{\max , 1}$ in Case 1 after applying mechanical load and shortly before unstable damage growth occurs. The maximum principal stress, $\sigma_{\max , 1}$, is largest where fibers are close together. However, these areas of high $\sigma_{\max , 1}$ do not correspond to the damaged areas as shown in figure 16(b) shortly before unstable damage growth occurs. Damage is largest at the boundary of the unit cell. The difference between the location of maximum damage and largest $\sigma_{\max , 1}$ has two reasons. First, damage initiation and progression is dependent on strain, not on stress. Secondly, by definition, areas of increased damage have softened and will only be able to hold a reduced amount of stress.

Figure 15(c) shows the maximum principal stress, $\sigma_{\max , 2}$, due to cure effects in Case 2 prior to applying external mechanical loads. The stress pattern is similar to the one that can be found for Case 1 in figure 15(a). However, due to larger cure shrinkage the amount of $\sigma_{\max , 2}$ is generally larger, than $\sigma_{\max , 1}$ at the end of cure. It can be seen from figure 16(c) that damage is already existent in the cured part, prior to applying external load. The location of the damage is identical to the one found in Case 1 after loading, as shown in figure 16(b). The maximum principle stress shortly before failure in Case 2 are shown in figure 15(d). Stresses are largest in the center of the fiber tow and where fibers are close together. Low $\sigma_{\max , 2}$ can be found towards the edge of the RUC. In this region significant amount of damage and softening took place, as shown in figure 16(d). Here the damage found before applying mechanical load has progressed. The damage volume and the magnitude of damage have increased.

To further investigate the evolution of stress and damage during cure, consider Point $\mathrm{A}$ as shown in figure 17(a). At this point damage already develops during cure in Case 2. The evolution of damage in Case 2 at Point $\mathrm{A}$ is depicted in figure 17(c). The figure shows the damage in the network that was created at the beginning of the curing process. To also characterize the total combined damage in all networks by a single value, a compound damage is introduced that weighs the damage in each network with the rate of 

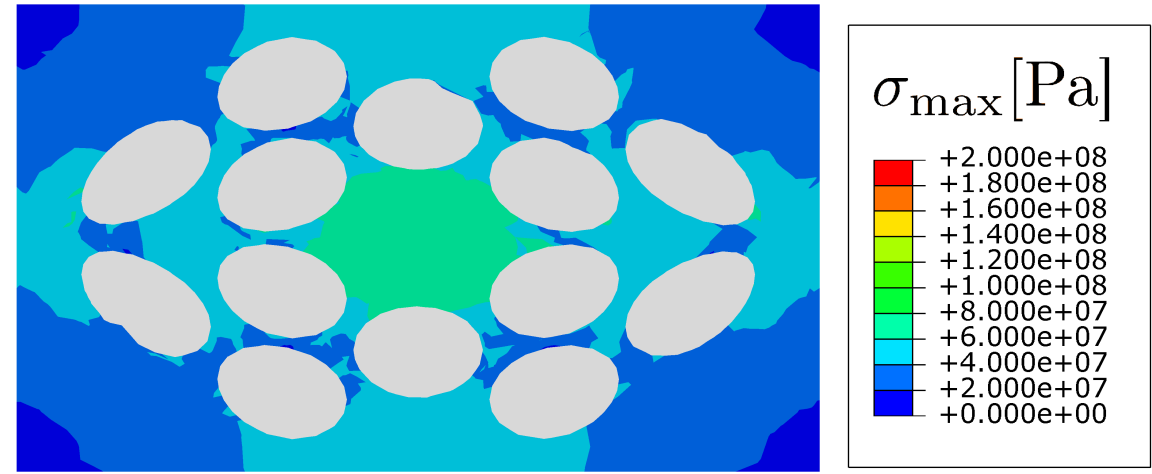

(a) Case 1, maximum principal stress at end of cure

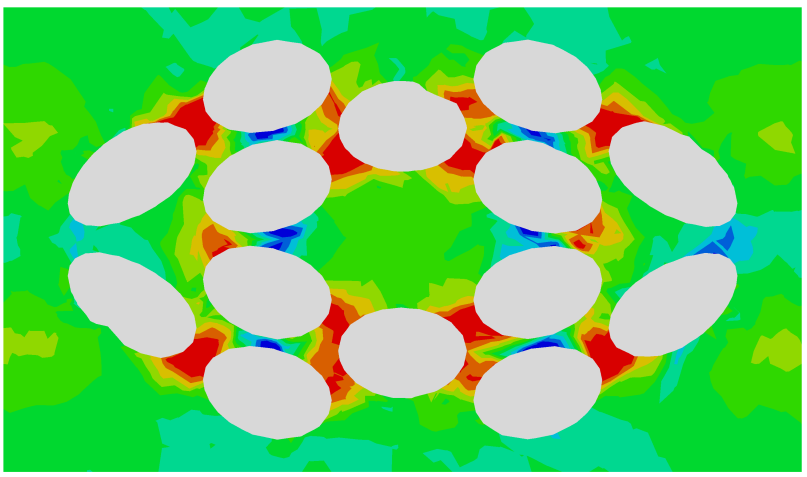

(b) Case 1, maximum principal stress at failure

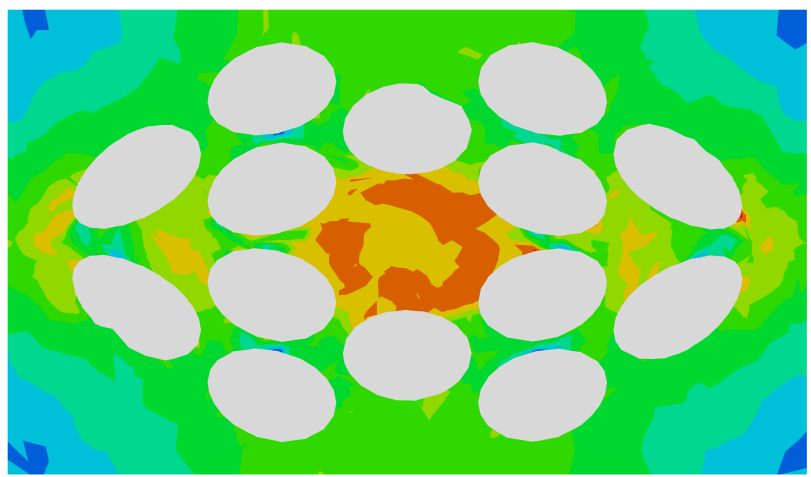

(c) Case 2, maximum principal stress at end of cure

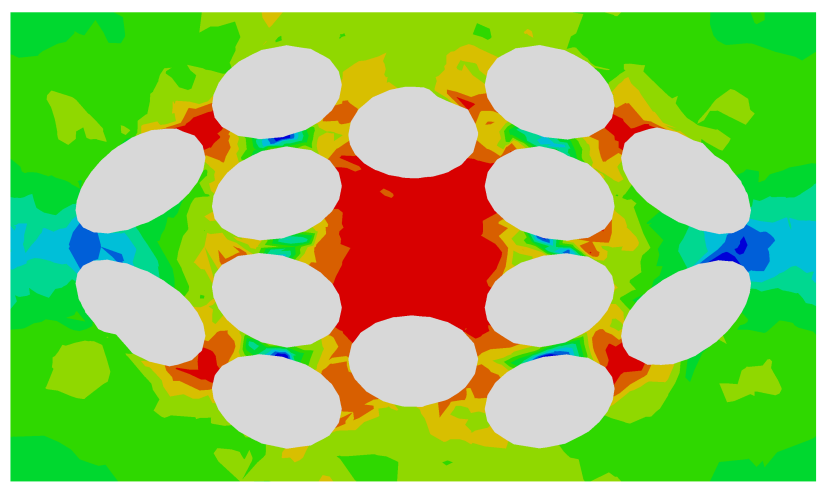

(d) Case 2, maximum principal stress at failure

Figure 15. Maximum principle stress in cross section $\square A B C D$ 

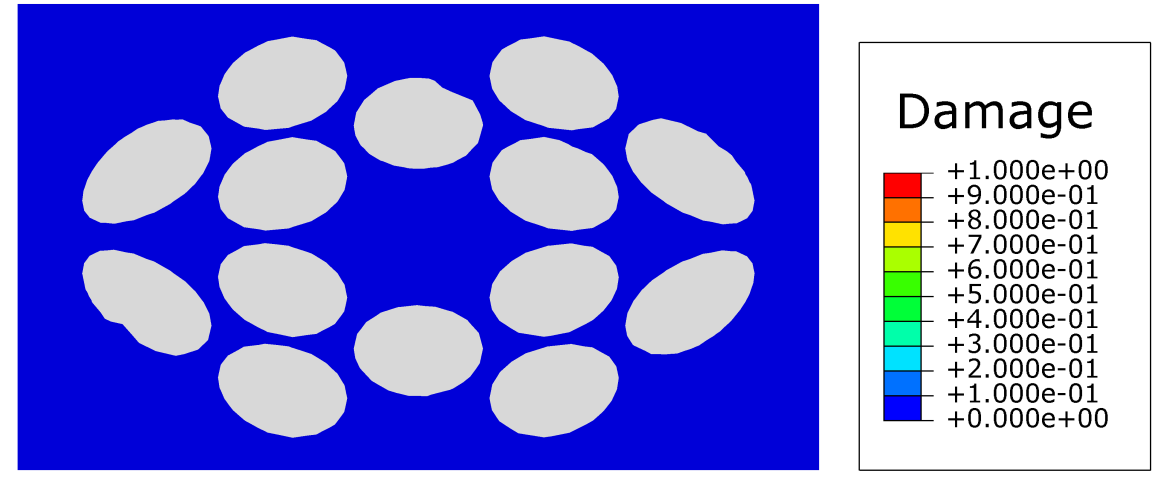

(a) Case 1, damage at end of cure

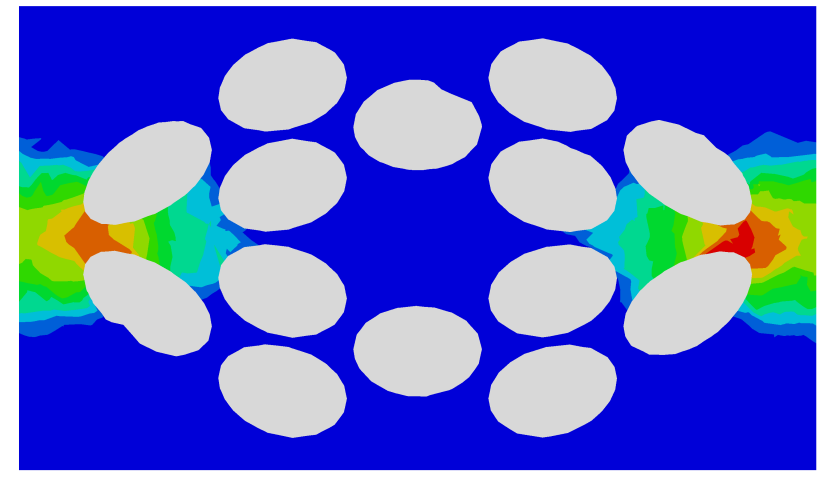

(b) Case 1, damage at failure

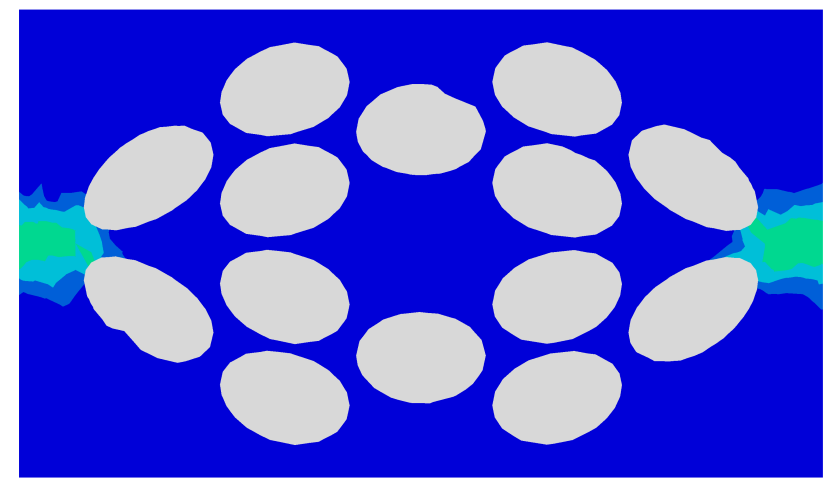

(c) Case 2, damage at end of cure

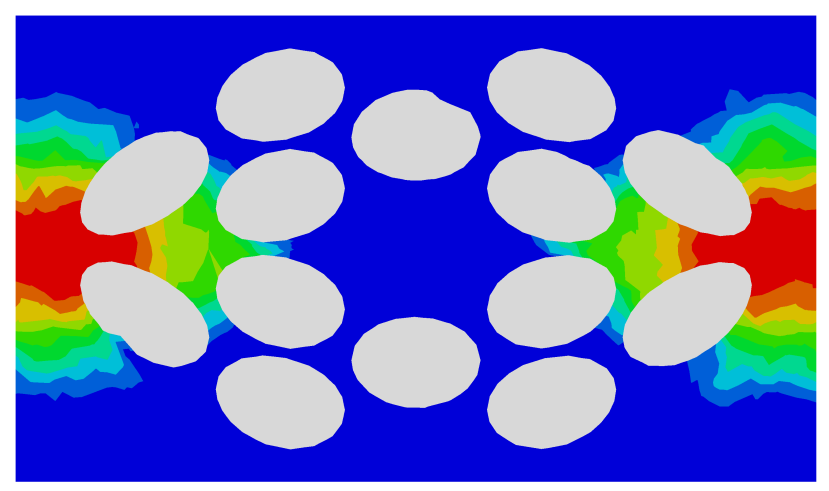

(d) Case 2, damage at failure

Figure 16. Damage in the first network, $\mathbf{D}(\mathbf{t}, \mathbf{0})$, in cross section $\square A B C D$ 
cure. Thus,

$$
D_{\text {tot }}(t)=\int_{0}^{t} \dot{\phi}(s) D(t, s) \mathrm{d} s
$$

The evolution of maximum principle stress is shown in figure 17(d). To properly interpret the results it is also necessary to consider the evolution of temperature and degree of cure at that point as shown in figure 17(b). The evolution of stress and damage can be divided into several stages. For the first 200 seconds no significant maximum principle stress can be seen due to vanishing shear modulus. At this point gelation occurs and the continued chemical shrinkage leads to increasing stresses. Due to slower curing rates at later times, as shown in figure 17(b), the creation of stresses also slows down. After 700 seconds the strains in the first network exceed damage onset strain. Continued cure shrinkage leads to an increase in damage. After 1800 seconds full cure is reached and no further increase in damage or maximum principle strain due to chemical effects can be observed. After 3600 seconds the temperature in the specimen is lowered. This leads to further damage and stress generation. Strictly speaking, if the cured epoxy was treated as a solid with stress-strain relations as described in figure 7, an increase in damage due to an increase in strain should be accompanied by a reduction in stress. This does not have to be the case in the curing of an epoxy. Networks created at different times have a different damage state. Therefore, while the networks created at the beginning accumulated a significant amount of damage, the networks created at the end continue to carry load. Generally it can be expected that networks created at the beginning show larger damage compared to networks created at later times, because they show the largest differential mechanical strain relative to their initial configuration. It should be noted that despite the sharp decrease of temperature in figure 17(b) after 3600 seconds, the increase of damage in figure 17(c) and maximum principal stress in figure 17(d) is spread out over the time from 3000 seconds to 4000 seconds. This due to the time discretization of the FE simulation. Smaller time steps will resolve the cooling down phase better, but the computational time will accordingly increase.

The findings of the 5 different cases can be compared with previous studies by Song ${ }^{1}$ who did similar tension tests in the laboratory for braided composite strips, in order to find the effictive ("in-situ") matrix material properties. He also, subsequently, investigated the compression response of a 2D triaxial braided textile carbon fiber and Hetron ${ }^{\mathrm{TM}}$ matrix composite. The fiber tows were modeled using the CCM model as a homogenization technique. The matrix material included plastic and damage effects, where the damage was closely related to the equivalent plastic strain, and calibrated against the "in-situ" matrix properties. The maximum stress with "virgin" properties, that do not account for residual stresses due to cure, is $700 \mathrm{MPa}$. The maximum stress with "in-situ" properties that include corrections for residual stresses is $300 \mathrm{MPa}$, thus leading to a reduction in maximum stress of $57 \%$.

The current study, as well as the one by Song ${ }^{1}$ aims to find the response of a braided composite in the presence of manufacturing induced stresses. In the calculations by Song, the stress state after complete cure was not known. Therefore, the effective "in-situ" material properties needed to be calibrated through coupon level experimental stress-strain curves. The aim of the current study is to provide an integrated computational framework that avoids such calibration procedures and instead directly captures the physical aspects of the composite manufacturing process to arrive at a unified and integrated framework to analyze the subsequent deformation response of textile composite structures.

\section{Conclusions}

An integrated computational materials science and engineering framework for textile composites has been presented. By understanding the role of the curing process of matrix materials (polymer resin) in textile composites and the influence of stresses generated during the curing process due to chemical shrinkage and thermal effects on subsequent performance of textile composites, it becomes possible to optimize the cure cycle so that the curing induced effects can be made to be beneficial in subsequent deformation response, under service loads. To accomplish this, several physical aspects needed to be accounted for, including thermal, chemical and mechanical effects. The evolution of temperature was coupled to a phenomenological curing model. Both effects depend strongly on each other and the corresponding equations were solved simultaneously.

A model was developed to describe the phase transition of a liquid epoxy resin and hardener mixture. The idea of continuously developing networks has been used. These networks interpenetrate each other, but do 


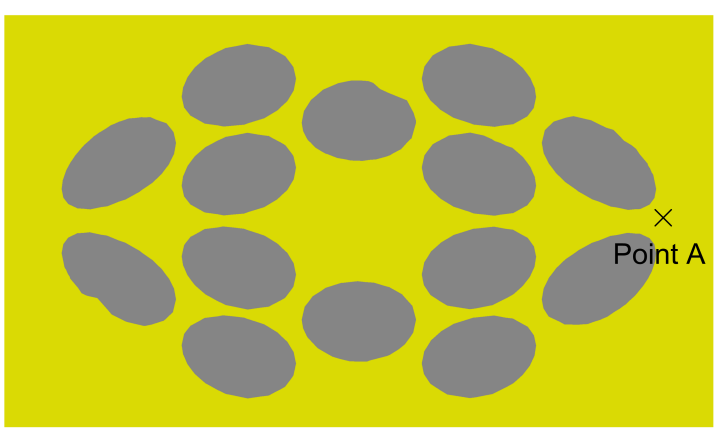

(a) Location where evolution stress and damage is investigated

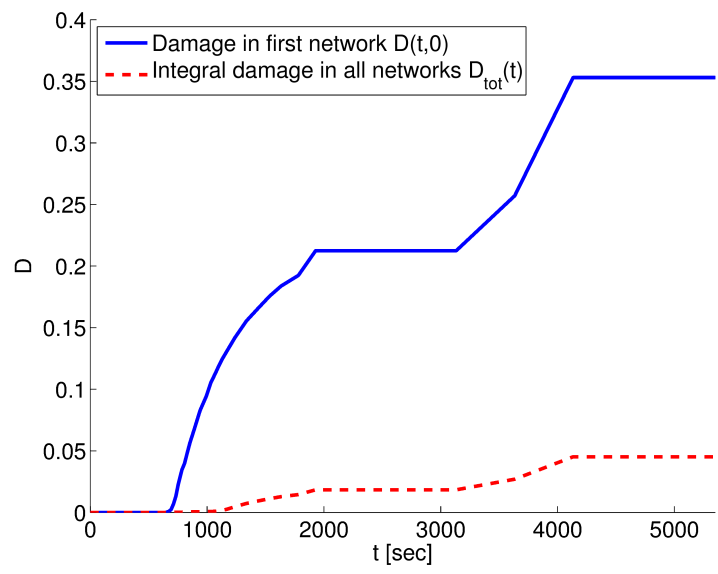

(c) Evolution of damage at Point A

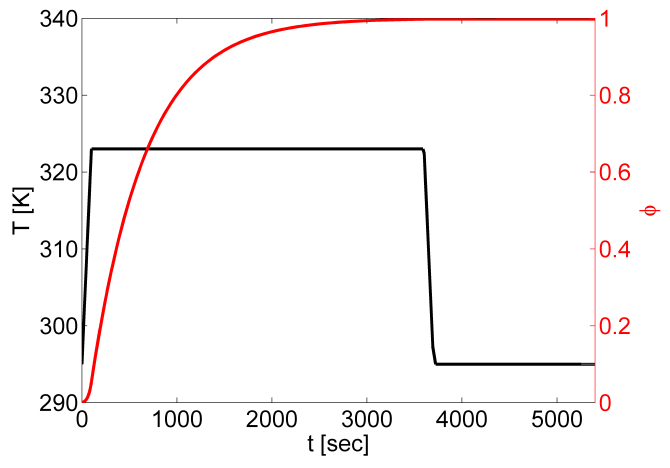

(b) Temperature and degree of cure at Point $\mathrm{A}$

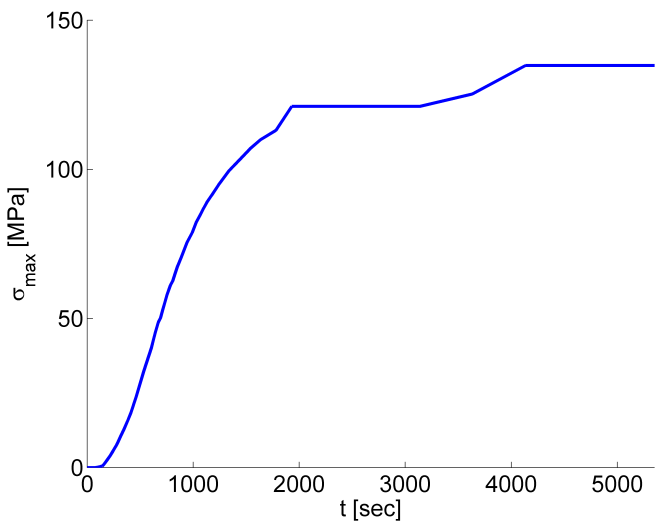

(d) Evolution of maximum principle stress at Point A 
not otherwise interact with each other. Different networks may have different thermal, elastic and inelastic properties and states. Especially, different networks have different stress free reference configurations. Due to thermal gradients in time and space and cure shrinkage of newly generated networks, stresses develop. These stresses may reduce (or increase) the margin of external loads that can be applied before the epoxy starts failing. The application of the network curing model has been shown for woven composites.

Inelastic response during and after cure was modeled using a nonlocal damage model that preserves mesh objectivity in finite element calculations. The damage state can be different for each network. The non-local damage model prevents the introduction of pathological mesh sensitivity in strain localization due to postpeak damage induced softening. Such effects take place in numerical simulations executed using the finite element method. The extended curing-damage model has been applied to a woven composite made of carbon fibers and epoxy matrix. The woven tows have been modeled using representative fibers, thus eliminating the need for a homogenization scheme of the curing and damaging fibers tows. For the representative unit cell used in this study, the effect of cure shrinkage and cure at different temperatures have been quantified and a reduction of the maximum applicable external load of over $30 \%$ for the given parameters was found for the case of tension response. The material properties of the curing model was derived from Brilliouin and Raman light scattering data. It was also shown how the material properties necessary to describe damage can be related to matrix fracture energy. A computational framework that integrates multiple physics effects in a unified and executable numerical framework is valuable for several engineering applications of textile composites.

\section{References}

${ }^{1}$ Song, S., Compression Response of Tri-axially Braided Textile Composites, Ph.D. thesis, University of Michigan, Ann Arbor, 2007.

${ }^{2}$ Song, S., Waas, A. M., Shahwan, K. W., Xiao, X., and Faruque, O., "Braided textile composites under compressive loads: Modeling the response, strength and degradation," Composites Science and Technology, Vol. 67, No. 15-16, 2007, pp. 30593070.

${ }^{3}$ Song, S., Waas, A. M., Shahwan, K. W., Faruque, O., and Xiao, X., "Compression Response of 2D Braided Textile Composites: Single Cell and Multiple Cell Micromechanics Based Strength Predictions," Journal of Composite Materials, Vol. 42, No. 23, 2008, pp. 2461-2482.

${ }^{4}$ Song, S., Waas, A. M., Shahwan, K. W., Faruque, O., and Xiao, X. S., "Compression response, strength and post-peak response of an axial fiber reinforced tow," International Journal of Mechanical Sciences, Vol. 51, No. 7, 2009 , pp. 491 - 499.

${ }^{5}$ Song, S., Waas, A. M., Shahwan, K. W., Faruque, O., and Xiao, X., "Effects of Matrix Microcracking on the Response of 2D Braided Textile Composites Subjected to Compression Loads," Journal of Composite Materials, Vol. 44, No. 2, 2010, pp. 221-240.

${ }^{6}$ Yerramalli, C. S. and Waas, A. M., "In Situ Matrix Shear Response Using Torsional Test Data of Fiber Reinforced Unidirectional Polymer Composites," Journal of Engineering Materials and Technology, Vol. 124, No. 2, 2002, pp. 152-159.

${ }^{7}$ Ramakrishnan, B., Zhu, L., and Pitchumani, R., "Curing of Composites Uning Internal Resistive Heating," Journal of Manufacturing Sciences and Egnieering, Vol. 122, 2000, pp. 124-131.

${ }^{8}$ Shanku, R., Vaughan, J., and Roux, J., "Rheological Characteristics and Cure Kinetics of EPON 862/W Epoxy Used in Pultrusion," Advances in Polymer Technology, Vol. 16, No. 4, 1997, pp. 297-311.

${ }^{9}$ O'Brien, D. J. and White, S. R., "Cure Kinetics, Gelation, and Glass Transition of Bisphenol F Epoxide," Polymer Engineering and Science, Vol. 43, No. 4, 2003, pp. 863-874.

${ }^{10}$ Xie, D. and Waas, A. M., "Discrete cohesive zone model for mixed-mode fracture using finite element analysis," Engineering Fracture Mechanics, Vol. 73, No. 13, 2006, pp. 1783 - 1796.

${ }^{11}$ Xie, D., Salvi, A. G., Sun, C., Waas, A. M., and Caliskan, A., "Discrete Cohesive Zone Model to Simulate Static Fracture in 2D Triaxially Braided Carbon Fiber Composites," Journal of Composite Materials, Vol. 40, No. 22, 2006, pp. 2025-2046.

${ }^{12}$ Chaboche, J.-L., "Continuous damage mechanics - A tool to describe phenomena before crack initiation," Nuclear Engineering and Design, Vol. 64, No. 2, 1981, pp. 233-247.

${ }^{13}$ Lemaitre, J. and Desmorat, R., Engineering Damage Mechanics: Ductile, Creep, Fatigue and Brittle Failures, SpringerVerlag, 2005.

${ }^{14}$ Lee, E. H., Rogers, T. G., and Woo, T. C., "Residual Stresses in a Glass Plate Cooled Symmetrically from Both Surfaces," Journal of the American Ceramic Society, Vol. 48, No. 9, 1965, pp. 480-487.

${ }^{15}$ Ishikawa, Takashi, C. T.-W., "One-dimensional micromechanical analysis of woven fabric composites," AIAA journal, Vol. 21, No. 12, 1983, pp. 1714-1721.

${ }^{16}$ Naik, N. and Shembekar, P., "Elastic Behavior of Woven Fabric Composites: Lamina Analysis," Journal of Composite Materials, Vol. 26, No. 15, 1992, pp. 2196-2225.

${ }^{17}$ Sankar, B. V. and Marrey, R. V., "A unit-cell model of textile composite beams for predicting stiffness properties," Composites Science and Technology, Vol. 49, No. 1, 1993, pp. $61-69$.

${ }^{18}$ Naik, N. and Ganesh, V., "Prediction of on-axes elastic properties of plain weave fabric composites," Composites Science and Technology, Vol. 45, No. 2, 1992, pp. $135-152$. 
${ }^{19}$ Zhang, Y. C. and Harding, J., "A numerical micromechanics analysis of the mechanical properties of a plain weave composite," Computers \& Structures, Vol. 36, No. 5, 1990, pp. 839 - 844.

${ }^{20}$ Karkkainen, R. L. and Sankar, B. V., "A direct micromechanics method for analysis of failure initiation of plain weave textile composites," Composites Science and Technology, Vol. 66, No. 1, 2006, pp. $137-150$.

${ }^{21}$ Tsai, S. W. and Hahn, H. T., Introduction to composite materials, Technomic Publishing Company, Inc., Lnacaster, PA, 1980

${ }^{22}$ Whitcomb, J. and Srirengan, K., "Effect of various approximations on predicted progressive failure in plain weave composites," Composite Structures, Vol. 34, No. 1, 1996, pp. 13 - 20.

${ }^{23}$ Quek, S. C., Waas, A. M., Shahwan, K. W., and Agaram, V., "Analysis of 2D triaxial flat braided textile composites," International Journal of Mechanical Sciences, Vol. 45, No. 6-7, 2003, pp. $1077-1096$.

${ }^{24}$ Quek, S. C., Waas, A., Shahwan, K. W., and Agaram, V., "Compressive response and failure of braided textile composites: Part 2-computations," International Journal of Non-Linear Mechanics, Vol. 39, No. 4, 2004, pp. 649 - 663.

${ }^{25}$ Hill, R., "A Theory of the Yielding and Plastic Flow of Anisotropic Metals," Proceedings of the Royal Society of London. Series A, Mathematical and Physical Sciences, Vol. 193, No. 1033, 1948, pp. pp. 281-297.

${ }^{26}$ Rabearison, N., Jochum, C., and Grandidier, J., "A FEM coupling model for properties prediction during the curing of an epoxy matrix," Computational Materials Science, Vol. 45, No. 3, 2009, pp. 715 - 724, Proceedings of the 17th International Workshop on Computational Mechanics of Materials - IWCMM-17.

${ }^{27}$ Corden, T. J., Jones, I. A., Jones, D. T., and Middleton, V., "The mechanisms of interlaminar cracking in thick resin transfer moulded composite cylinders," Composites Part A: Applied Science and Manufacturing, Vol. 29, No. 4, 1998, pp. 455 -464 .

${ }^{28}$ Plepys, A. and Farris, R., "Evolution of residual stresses in three-dimensionally constrained epoxy resins," Polymer, Vol. 31, No. 10, 1990, pp. $1932-1936$.

${ }^{29}$ Chekanov, Y. A., Korotkov, V. N., Rozenberg, B. A., Dhzavadyan, E. A., and Bogdanova, L. M., "Cure shrinkage defects in epoxy resins," Polymer, Vol. 36, No. 10, 1995, pp. $2013-2017$.

${ }^{30}$ Darrow, D. A. and Smith, L. V., "Isolating Components of Processing Induced Warpage in Laminated Composites," Journal of Composite Materials, Vol. 36, No. 21, 2002, pp. 2407-2419.

${ }^{31}$ Fernlund, G., Rahman, N., Courdji, R., Bresslauer, M., Poursartip, A., Willden, K., and Nelson, K., "Experimental and numerical study of the effect of cure cycle, tool surface, geometry, and lay-up on the dimensional fidelity of autoclave-processed composite parts," Composites Part A: Applied Science and Manufacturing, Vol. 33, No. 3, 2002, pp. 341 - 351.

${ }^{32}$ Albert, C. and Fernlund, G., "Spring-in and warpage of angled composite laminates," Composites Science and Technology, Vol. 62, No. 14, 2002, pp. 1895 - 1912.

${ }^{33}$ Johnston, A., Vaziri, R., and Poursartip, A., "A Plane Strain Model for Process-Induced Deformation of Laminated Composite Structures," Journal of Composite Materials, Vol. 35, No. 16, 2001, pp. 1435-1469.

${ }^{34}$ Kim, J. W., Lee, J. H., Kim, H. G., Kim, H. S., and Lee, D. G., "Reduction of residual stresses in thick-walled composite cylinders by smart cure cycle with cooling and reheating," Composite Structures, Vol. 75, No. 1-4, 2006, pp. 261 266, Thirteenth International Conference on Composite Structures - ICCS/13.

${ }^{35} \mathrm{Kim}, \mathrm{K}$. and Hahn, H., "Residual stress development during processing of graphite/epoxy composites," Composites Science and Technology, Vol. 36, No. 2, 1989, pp. $121-132$.

${ }^{36}$ White, S. and Hahn, H., "Cure Cycle Optimization for the Reduction of Processing-Induced Residual Stresses in Composite Materials," Journal of Composite Materials, Vol. 27, No. 14, 1993, pp. 1352-1378.

${ }^{37}$ Shrotriya, P., Sottos, N. R., and Skipor, A. F., "Residual Stress Development during Relamination of Woven Composite Circuit Boards," Journal of Composite Materials, Vol. 35, No. 10, 2001, pp. 905-927.

${ }^{38}$ Capehart, T., Muhammad, N., and Kia, H. G., "Compensating Thermoset Composite Panel Deformation using Corrective Molding," Journal of Composite Materials, Vol. 41, No. 14, 2007, pp. 1675-1701.

${ }^{39}$ Kamal, M. R., "Thermoset Characterization for Moldability Analysis," Polymer Engineering and Science, Vol. 14, No. 3, 1974, pp. 231-239.

${ }^{40}$ Plepys, A., Vratsanos, M. S., and Farris, R. J., "Determination of residual stresses using incremental linear elasticity," Composite Structures, Vol. 27, No. 1-2, 1994, pp. 51 - 56, Special Issue Advances in Fiber Reinforced Composites Technology.

${ }^{41}$ Bogetti, T. A. and Gillespie, J. W., "Process-Induced Stress and Deformation in Thick-Section Thermoset Composite Laminates," Journal of Composite Materials, Vol. 26, No. 5, 1992, pp. 626-660.

${ }^{42}$ Adolf, D. B., Martin, J. E., Chambers, R. S., Burchett, S. N., and Guess, T. R., "Stresses during thermoset cure," Journal of Materials Research, Vol. 13, 1998, pp. 530-550.

${ }^{43}$ Adolf, D. and Chambers, R., "Verification of the capability for quantitative stress prediction during epoxy cure," Polymer, Vol. 38, 1997, pp. 5481-5490.

${ }^{44}$ White, S. and Hahn, H., "Process Modeling of Composite Materials: Residual Stress Development during Cure. Part I. Model Formulation," Journal of Composite Materials, Vol. 26, No. 16, 1992, pp. 2402-2422.

${ }^{45}$ Lange, J., Toll, S., Mnson, J.-A. E., and Hult, A., "Residual stress build-up in thermoset films cured above their ultimate glass transition temperature," Polymer, Vol. 36, No. 16, 1995, pp. 3135 - 3141.

${ }^{46}$ Adolf, D. B. and Chambers, R. S., "A thermodynamically consistent, nonlinear viscoelastic approach for modeling thermosets during cure," Journal of Rheology, Vol. 51, No. 1, 2007, pp. 23-50.

${ }^{47}$ Adolf, D. B., Chambers, R. S., and Caruthers, J. M., "Extensive validation of a thermodynamically consistent, nonlinear viscoelastic model for glassy polymers," Polymer, Vol. 45, No. 13, 2004, pp. $4599-4621$.

${ }^{48}$ Mai, Y., Yee, A. F., Wineman, A. S., and Xiao, C., "Streff evolution during thermoset cure," Material Research Society Symposia Proceedings, Vol. 515, 1998.

${ }^{49}$ Mei, Y., Stress Evolution in a Conductive Adhesive during Curing and Cooling, Ph.D. thesis, University of Michigan, Ann Arbor, Michigan, 2000. 
${ }^{50} \mathrm{Li}$, M., Zhu, Q., Geubelle, P. H., and Tucker III, C. L., "Optimal curing for thermoset matrix composites: Thermochemical considerations," Polymer Composites, Vol. 22, No. 1, 2001, pp. $118-131$.

${ }^{51}$ Gopal, A. K., Adali, S., and Verijenko, V. E., "Optimal temperature profiles for minimum residual stress in the cure process of polymer composites," Composite Structures, Vol. 48, No. 1-3, 2000, pp. 99 - 106.

${ }^{52} \mathrm{Zhu}$, Q. and Geubelle, P. H., "Dimensional Accuracy of Thermoset Composites: Shape Optimization," Journal of Composite Materials, Vol. 36, No. 6, 2002, pp. 647-672.

${ }^{53}$ Fung, Y., A First Course in Continuum Mechanics, Prentence Hall, Englewood Cliffs, 3rd ed., 1994.

${ }^{54}$ Doghri, I., Mechanics of Deformable Solids, Springer Verlag, Berlin, 2000.

${ }^{55}$ Ramos, J., Pagani, N., Riccardi, C., Borrajo, J., Goyanes, S., and Mondragon, I., "Cure kinetics and shrinkage model for epoxy-amine systems," Polymer, Vol. 46, No. 10, 2005, pp. 3323 - 3328.

${ }^{56}$ Beyer, M. K. and Clausen-Schaumann, H., "Mechanochemistry:The Mechanical Activation of Covalent Bonds," Chemical Reviews, Vol. 105, 2005, pp. $2921-2948$.

${ }^{57}$ Ikegawa, N., Hamada, H., and Maekawa, Z., "Effect of compression process on void behavior in structural resin transfer molding," Polymer Engineering \& Science, Vol. 36, No. 7, 1996, pp. 953-962.

${ }^{58}$ Rudd, C., ASM Handbook, Vol. 21-Composites, chap. Resin transfer modling and strutural reaction injection molding, ASM International, 2001, pp. 493-500.

${ }^{59}$ Revello, M., Saggese, L., and Gaiero, E., Comprehensive Composite Materials, chap. Compression Molding of SMCs, Elsevier, Amsterdam, 2000, pp. 763-805.

${ }^{60}$ Hossain, M., Possart, G., and Steinmann, P., "A small-strain model to simulate the curing of thermosets," Computational Mechanics, Vol. 43, 2009, pp. $769-779$.

${ }^{61}$ Salvi, A. and Waas, A. M., "Report to the Automotive Composite Consortium: Static and Dynamic Fracture of Carbon Fiber Reinforced Braided Composites," Tech. rep., University of Michigan, January 2005.

${ }^{62}$ de Souza Neto, E. A., Perić, D., and Owen, D. R. J., Computational Methods for Plasticity: Theory and Applications, John Wiley \& Sons, Ltd, 2008.

${ }^{63}$ Kachanov, L., "Rupture Time Under Creep Conditions," International Journal of Fracture, Vol. 97, 1999, pp. 11-18, 10.1023/A:1018671022008.

${ }^{64}$ Rabotnov, Y., "On the Equations of State for Creep," Proceedings of the Institution of Mechanical Engineers, Conference Proceedings 1964-1970 (vols 178-184), Various titles labelled Volumes A to S, pp. 117-122.

${ }^{65}$ Leckie, F. and Hayhurst, D., "Constitutive equations for creep rupture," Acta Metallurgica, Vol. 25, No. 9, 1977, pp. 1059 -1070 .

${ }^{66}$ Lemaitre, J. and Chaboche, J.-L., Mechanics of Solid Materials, Cambridge University Press, 1994.

${ }^{67}$ Lemaitre, J., "A Continuous Damage Mechanics Model for Ductile Fracture," Journal of Engineering Materials and Technology, Vol. 107, No. 1, 1985, pp. 83-89.

${ }^{68}$ Jirásek, M. and Bažant, Z. P., Inelastic Analysis of Structures, John Wiley \& Sons, Ltd, 2002.

${ }^{69} \mathrm{de}$ Borst, R., "Computation of post-bifurcation and post-failure behavior of strain-softening solids," Computers $\mathbb{E}$ Structures, Vol. 25, No. 2, 1987, pp. 211 - 224.

${ }^{70}$ Bažant, Z. and Oh, B., "Crack band theory for fracture of concrete," Materials and Structures, Vol. 16, 1983, pp. 155$177,10.1007 /$ BF02486267.

${ }^{71}$ Peerlings, R. H. J., Geers, M. G. D., de Borst, R., and Brekelmans, W. A. M., "A critical comparison of nonlocal and gradient-enhanced softening continua," International Journal of Solids and Structures, Vol. 38, No. 44-45, 2001, pp. 7723 7746 .

${ }^{72}$ Bažant, Z., "Imbricate Continuum and its Variational Derivation," Journal of Engineering Mechanics, Vol. 110, 1984, pp. 1693-1712.

${ }^{73}$ Pijaudier-Cabot, G. and Bažant, Z. P., "Nonlocal Damage Theory," Journal of Engineering Mechanics, Vol. 113, 1987, pp. $1512-1533$.

${ }^{74}$ M H.-B., "A variational principle for gradient plasticity," International Journal of Solids and Structures, Vol. 28, No. 7, 1991, pp. $845-857$.

${ }^{75}$ Lasry, D. and Belytschko, T., "Localization limiters in transient problems," International Journal of Solids and Structures, Vol. 24, No. 6, 1988, pp. $581-597$.

${ }^{76}$ Peerlings, R., de Borst, R., Brekelmans, W. A. M., and de Vree, J. H. P., "Gradient enhanced damage for quasi-brittle materials," International Journal for Numerical Methods in Engineering, Vol. 39, 1996, pp. 3391-3403.

${ }^{77}$ Dorgan, R. J., A nonlocal model for coupled damage-plasticity incorporating gradients of internal state variables at multiscales, Ph.D. thesis, Louisiana State University, Baton Rouge, Louisiana.

${ }^{78}$ Askes, H., Pamin, J., and de Borst, R., "Dispersion analysis and element-free Galerkin solutions of second- and fourthorder gradient-enhanced damage models," International Journal for Numerical Methods in Engineering, Vol. $49,2000$.

${ }^{79}$ Askes, H. and Sluys, L. J., "Explicit and implicit gradient series in damage mechanics," European Journal of Mechanics - A/Solids, Vol. 21, No. 3, 2002, pp. 379 - 390.

${ }^{80}$ Dorgan, R. J. and Voyiadjis, G. Z., "A Mixed Finite Element Implementation of a Gradient-enhanced Coupled Damage Plasticity Model," International Journal of Damage Mechanics, Vol. 15, No. 3, 2006, pp. 201-235.

${ }^{81}$ Geers, M. G. D., Ubachs, R. L. J. M., and Engelen, R. A. B., "Strongly non-local gradient-enhanced finite strain elastoplasticity," International Journal for Numerical Methods in Engineering, Vol. 56, 2003, pp. 2039-2068.

${ }^{82}$ Engelen, R. A. B., Geers, M. G. D., and Baaijens, F. P. T., "Nonlocal implicit gradient-enhanced elasto-plasticity for the modelling of softening behaviour," International Journal of Plasticity, Vol. 19, No. 4, 2003, pp. 403 - 433.

${ }^{83}$ Challamel, N., "A variationally based nonlocal damage model to predict diffuse microcracking evolution," International Journal of Mechanical Sciences, Vol. 52, No. 12, 2010, pp. 1783 - 1800. 
${ }^{84}$ Chike, K. E., Myrick, M. L., Lyon, R. E., and Angel, S. M., "Raman and Near-Infrared Studies of an Epoxy Resin," Appl. Spectrosc., Vol. 47, No. 10, 1993, pp. 1631-1635.

${ }^{85}$ Merad, L., Cochez, M., Margueron, S., Jauchem, F., Ferriol, M., Benyoucef, B., and Bourson, P., "In-situ monitoring of the curing of epoxy resins by Raman spectroscopy," Polymer Testing, Vol. 28, No. 1, 2009, pp. $42-45$.

${ }^{86}$ Yamura, H., Matsukawa, M., Otani, T., and Ohtori, N., "Brillouin Scattering Study on the Elastic Properties of Epoxy Adhesive Layer," Japanese Journal of Applied Physics, Vol. 38, No. Part 1, No. 5B, 1999, pp. 3175-3178.

${ }^{87}$ O'Brian, D. J., Mather, P. T., and White, S. R., "Viscoelastic Properties of an Epoxy Resin during Cure," Journal of Composite Materials, Vol. 35, 2001, pp. 883 - 904.

${ }^{88} \mathrm{Li}, \mathrm{C} .$, Potter, K., Wisnom, M. R., and Stringer, G., "In-situ measurement of chemical shrinkage of MY750 epoxy resin by a novel gravimetric method," Composites Science and Technology, Vol. 64, No. 1, 2004, pp. 55 - 64 .

${ }^{89}$ Lange, J., Toll, S., Månson, J.-A. E., and Hult, A., "Residual stress build-up in thermoset films cured below their ultimate glass transition temperature," Polymer, Vol. 38, No. 4, 1997, pp. $809-815$.

${ }^{90}$ Yates, B., McCalla, B. A., Phillips, L. N., Kingston-Lee, D. M., and Rogers, K. F., "The thermal expansion of carbon fibre-reinforced plastics," Journal of Materials Science, Vol. 14, 1979, pp. 1207-1217, 10.1007/BF00561306.

${ }^{91}$ Bujard, P., Kuhnlein, G., Ino, S., and Shiobara, T., "Thermal conductivity of molding compounds for plastic packaging," Electronic Components and Technology Conference, 1994. Proceedings., 44th, May 1994, pp. 159 -163.

${ }^{92}$ Hexion Specialty Chemicals, Inc., "Diglycidyl Ether of Bisphenol F (DGEBF) - Performance Properties," .

${ }^{93}$ Varshney, V., Patnaik, S. S., Roy, A. K., and Farmer, B. L., "Molecular Dynamics Study of Thermal Transport Phenomena in Cross-linked Polymer: Epon 862 with Curing Agent W (DETDA)," .

${ }^{94}$ Schapery, R., "Mechanical characterization and analysis of inelastic composite laminates with growing damage," Proceedings of the Third ASCE/ASME Joint Mechanics Conference, San Diego, July 1989.

${ }^{95}$ Schapery, R., "A theory of mechanical behavior of elastic media with growing damage and other changes in structure," Journal of the Mechanics and Physics of Solids, Vol. 38, No. 2, 1990, pp. $215-253$.

${ }^{96}$ Schapery, R. and Sicking, S., "On nonlinear constitutive equations for elastic and viscoelastic composites with growing damage," Mechanical behavior of materials; invited papers presentet at the Seventh International Conference on Mechanical Behavior of Materials - ICM7, 1995.

${ }^{97}$ Chen, C., Justice, R. S., Schaefer, D. W., and Baur, J. W., "Highly dispersed nanosilica-epoxy resins with enhanced mechanical properties," Polymer, Vol. 49, No. 17, 2008, pp. $3805-3815$.

${ }^{98}$ Zunjarrao, S. C. and Singh, R. P., "Characterization of the fracture behavior of epoxy reinforced with nanometer and micrometer sized aluminum particles," Composites Science and Technology, Vol. 66, No. 13, 2006, pp. 2296 - 2305.

${ }^{99}$ Simulia, ABAQUS 6.9 manual, Dassault Systems, 2009.

${ }^{100}$ C.Heinrich, Aldridge, M., Wineman, A., Kieffer, J., and Waas, A., "The influence of fiber packing on the homogenized response of cured fiber composites," 25th Annual Technical Conference American Society for Composites, 2010.

${ }^{101}$ Geers, M. G. D., de Borst, R., Brekelmans, W. A. M., and Peerlings, R. H. J., "Strain-based transient-gradient damage model for failure analyses," Computer Methods in Applied Mechanics and Engineering, Vol. 160, No. 1-2, 1998, pp. 133 - 153.

${ }^{102}$ Riks, E., "The Application of Newton's Method to the Problem of Elastic Stability," Journal of Applied Mechanics, Vol. 39, No. 4, 1972, pp. 1060-1065.

${ }^{103}$ Riks, E., "An incremental approach to the solution of snapping and buckling problems," International Journal of Solids and Structures, Vol. 15, No. 7, 1979, pp. $529-551$. 